\title{
Moduli of Sheaves Supported on Quartic Space Curves
}

\author{
Jinwon Choi, Kiryong Chung, \& Mario Maican
}

\begin{abstract}
As a continuation of the work of Freiermuth and Trautmann, we study the geometry of the moduli space of stable sheaves on $\mathbb{P}^{3}$ with Hilbert polynomial $4 m+1$. The moduli space has three irreducible components whose generic elements are, respectively, sheaves supported on rational quartic curves, on elliptic quartic curves, or on planar quartic curves. The main idea of the proof is to relate the moduli space with the Hilbert scheme of curves by wall crossing. We present all stable sheaves contained in the intersections of the three irreducible components. We also classify stable sheaves by means of their free resolutions.
\end{abstract}

\section{Introduction}

\subsection{Motivations and Results}

For a fixed polynomial $P(m)$ with rational coefficients, it is well known that the space parameterizing Gieseker-stable sheaves on a smooth projective variety $X$ with Hilbert polynomial $P(m)$ is a projective scheme ([30]). In this paper, we are specifically interested in the case where $P(m)=d m+\chi$ is a linear polynomial, as the moduli space is closely related to the relative Jacobian of families of curves. When $X$ is the complex projective plane $\mathbb{P}^{2}$, Le Potier [21] showed that the moduli space is an irreducible projective variety of dimension $d^{2}+1$, that it is locally factorial, and that its Picard group is generated by two explicitly constructed divisors. When the leading coefficient $d$ is small, the moduli spaces have been studied by many authors. Stratifications in terms of the free resolution of sheaves $[11 ; 24$; 25], topological invariants such as Poincaré polynomials [8; 5; 7; 37], stable base locus decompositions [7] are known.

In this paper we study the case where $X$ is $\mathbb{P}^{3}$. Let $\mathrm{M}(P(m))$ denote the moduli space of stable sheaves on $\mathbb{P}^{3}$ with Hilbert polynomial $P(m)$. Freiermuth and Trautmann [12] examined $\mathrm{M}(3 m+1)$. They gave a complete classification of sheaves in $\mathrm{M}(3 m+1)$ and proved that this moduli space has two irreducible components intersecting transversally. The first component, which we denote by $R_{3}$, parameterizes the structure sheaves of twisted cubic curves. The second component parameterizes the sheaves of the form $\mathcal{O}_{C}(p)$, where $C$ is a planar cubic curve, and $p \in C$.

Received June 19, 2015. Revision received January 13, 2016.

J. Choi was supported by the Sookmyung Women's University Research Grants 1-1503-0233.

K. Chung was partly supported by Korea NRF grant 2013R1A1A2006037. 
From the point of view of the birational geometry, $R_{3}$ is related to the moduli space of twisted cubic curves. Let $\bar{M}_{0}\left(\mathbb{P}^{r}, d\right)$ be the moduli space of genus zero stable maps to $\mathbb{P}^{r}$ of degree $d$. It is known that this is a projective normal variety containing a Zariski dense open subset consisting of irreducible rational curves of degree $d$. Specially, if $r=3$ and $d=3$, then the moduli space $\bar{M}_{0}\left(\mathbb{P}^{3}, 3\right)$ of stable maps is the unique flipping space of the component $R_{3}$ over the (normalization of the) Chow variety (see [2]). We remark that the flipping locus is exactly the locus of multiple covers of its image or the stable sheaves supported on nonreduced curves. More generally on $\mathbb{P}^{r}$, Chen, Coskun, and Crissman [3] proved that the moduli space of stable maps $\bar{M}_{0}\left(\mathbb{P}^{r}, 3\right)$ is one of the flip models of the compactified space of stable sheaves supported on rational curves. On the other hand, in [ 9 ; $10]$, by extending the flip map between the compactified moduli spaces of rational curves the authors established an explicit birational relation between $\bar{M}_{0}(X, 3)$ and $R_{3}(X)$ when $X$ is a projective homogenous variety, which is a natural generalization of the projective space. Until now, to the knowledge of the authors, there are no such results for higher degrees $d$. Thus, our project can be regarded as a starting point of understanding the various compactified moduli spaces of curves in $\mathbb{P}^{r}$.

In this paper we investigate $\mathrm{M}(4 m+1)$.

\section{DEFINITION 1.1.}

- The dual of a one-dimensional sheaf $F$ on $\mathbb{P}^{3}$ is defined by $F^{\mathrm{D}}=\mathcal{E} x t^{2}\left(F, \omega_{\mathbb{P}^{3}}\right)$.

- We call a sheaf $F$ planar if $\left.F \simeq F\right|_{H}$ for some plane $H \subset \mathbb{P}^{3}$ or, equivalently, the schematic support of $F$ is contained in $H$.

Our main result is that the space $\mathrm{M}(4 m+1)$ consists of three irreducible components.

THEOREM 1.2. Let $\mathrm{M}(4 m+1)$ be the moduli space of stable sheaves in $\mathbb{P}^{3}$ with Hilbert polynomial $4 m+1$. Then $\mathrm{M}(4 m+1)$ consists of three irreducible components whose general points are

(1) $\mathbf{R}$ : the structure sheaves of rational quartic curves;

(2) $\mathbf{E}$ : the dual sheaves $I_{p, C}^{\mathrm{D}}$ of the ideal sheaves $I_{p, C}$ of points $p$ on elliptic quartic curves $C$;

(3) P: the planar sheaves.

The component $\mathbf{R}$ is the compactification of the space of rational quartic curves that is predicted as a birational flip model of the space of finite maps (see [4]). The general member of $\mathbf{E}$ is a line bundle of the form $\mathcal{O}_{C}(p)$, where $p \in C$, for a smooth elliptic quartic curve $C$. Since $\operatorname{Ext}^{1}\left(\mathbb{C}_{p}, \mathcal{O}_{C}\right)=\mathbb{C}$, this line bundle fits into the unique nonsplit extension

$$
0 \rightarrow \mathcal{O}_{C} \rightarrow \mathcal{O}_{C}(p) \rightarrow \mathbb{C}_{p} \rightarrow 0 .
$$

Using the results in [1], we can easily see that $\mathbf{E}$ is birational to the universal elliptic quartic curve (for detail, see Lemma 3.5). As the supports of sheaves in $\mathbf{E}$ degenerate to singular quartic curves of arithmetic genus 1 , two complications arise. 
Firstly, the dimension of $\operatorname{Ext}^{1}\left(\mathbb{C}_{p}, \mathcal{O}_{C}\right)$ may jump to 2 (Lemma 3.3). Secondly, some extension sheaves may fail to be stable because they may have a destabilizing subsheaf that is isomorphic to a structure sheaf $\mathcal{O}_{L}$ of a line $L$, which is given by the push-out of $\operatorname{Ext}^{1}\left(\mathbb{C}_{p}, \mathcal{O}_{L}(-1)\right)=\mathbb{C}$, where we have an exact sequence

$$
0 \rightarrow \mathcal{O}_{L}(-1) \rightarrow \mathcal{O}_{C} \rightarrow \mathcal{O}_{C_{0}} \rightarrow 0
$$

for some planar cubic curve $C_{0}$. We overcome these difficulties by regarding such a locus as a bundle space over other base spaces.

The main idea of the proof of Theorem 1.2 is to relate $\mathrm{M}(4 m+1)$ with the Hilbert scheme of degree 4 curves by using wall crossing, which we review in Section 2. It is known that this Hilbert scheme consists of four irreducible components. One component consists of curves corresponding to unstable sheaves and is irrelevant for our purposes. The other three components are modified by wall crossing into $\mathbf{R}, \mathbf{E}$, and $\mathbf{P}$.

In Section 5 we describe the intersections of $\mathbf{R}, \mathbf{E}$, and $\mathbf{P}$ by using the elementary modifications of sheaves. The sheaves in $\mathbf{R} \cap \mathbf{P}$ and $\mathbf{E} \cap \mathbf{P}$ can be classified by using the description of the incident variety of the planar quartic curves with points (concretely, the relative Hilbert scheme); see [5]. The intersection $\mathbf{R} \cap \mathbf{E}$ is more complicated. A natural candidate for the general element in the intersection is provided by a pair of a nondegenerate singular elliptic curve and its singular point. The key issue is to prove that every degeneration of stable sheaves in $\mathbf{R} \cap \mathbf{E}$ is a limit of such pairs. It seems hard to describe the boundary of $\mathbf{R}$ in the Hilbert scheme, so, instead, we use the modification technique developed in $[9 ; 10]$. The authors in $[9 ; 10]$ compare the moduli space of stable maps (or finite maps) with the moduli space of stable sheaves when the degree of the maps (or sheaves) is at most 3. Starting from the canonical family of pure sheaves obtained as direct images of stable maps, they extend the birational maps into the birational regular maps after performing modifications of sheaves several times along the exceptional divisors of blowing-ups of the stable maps space. In our case of degree 4 , even though we do not have a full picture of the blowing-ups and modifications of sheaves, we can find the normal direction of the space of stable maps that provides the indicated stable sheaves. By combining with the computation of the deformation space we show that $\mathbf{R} \cap \mathbf{E}$ is a single irreducible divisor in $\mathbf{R}$.

For completeness, we present in the last section the possible free resolutions of the sheaves in $\mathrm{M}(4 m+1)$. These can be used in the local analysis of each component in a forthcoming work.

\section{Review of the Wall Crossing}

\subsection{General Framework}

In this section we review the wall crossing technique that we will use in the paper. Motivated by the Donaldson-Thomas/Pandharipande-Thomas correspondence [28], Stoppa and Thomas [31] studied a GIT wall-crossing between the Hilbert scheme of curves and the moduli space of stable pairs. A stable pair here is a pair of a one-dimensional sheaf and a section that generates the sheaf away 
from finitely many points. Both of the Hilbert scheme and the moduli space of stable pairs are equipped with a perfect obstruction theory, and hence the virtual invariants are defined by integrating the cohomology cycle against the virtual fundamental classes $([33 ; 28])$. Stoppa and Thomas realized both spaces as GIT quotients of a certain space of pairs, and by altering the linearization they showed that these two moduli spaces are related by GIT wall crossing.

We can go further. In [5], the authors study the wall-crossing for the moduli space $\mathrm{M}^{\alpha}(P(m))$ of $\alpha$-stable pairs, where $\alpha$ is a nonnegative rational number.

\section{DEFINITION 2.1.}

(1) A pair is a pair of a sheaf and a nonzero section.

(2) A sheaf $F$ is pure if for every nonzero subsheaf $G \subset F$, the dimension of the support of $G$ is the same as the dimension of the support of $F$.

(3) A pair $(s, F)$ is called $\alpha$-semistable if

(a) $F$ is a pure sheaf and

(b) for any proper nonzero subsheaf $F^{\prime} \subset F$,

$$
\frac{\chi\left(F^{\prime}(m)\right)+\delta \cdot \alpha}{r\left(F^{\prime}\right)} \leq \frac{\chi(F(m))+\alpha}{r(F)}
$$

for $m \gg 0$. Here $r(F)$ is the leading coefficient of the Hilbert polynomial $\chi(F(m))$, and $\delta=1$ if the section $s$ factors through $F^{\prime}$ and $\delta=0$ otherwise.

When the strict inequality holds, $(s, F)$ is called $\alpha$-stable. If a pair is $\alpha$ semistable but not $\alpha$-stable, then it is called strictly $\alpha$-semistable.

A pair is a special case of the coherent system by Le Potier [22] (see also [17]). A coherent system is a pair of a sheaf with a subspace $V$ of $H^{0}(F)$. So, a pair is a coherent system when the dimension of $V$ is one. We will use the notation $(1, F)$ to denote the pair of the sheaf $F$ with its nonzero section and $(0, F)$ when the section is taken to be zero. Although by definition the section in a pair must be nonzero, these notation is convenient when we study the $\alpha$-stability. The short exact sequence

$$
0 \rightarrow(1, G) \rightarrow(1, F) \rightarrow(0, F / G) \rightarrow 0
$$

indicates that $G$ is a subsheaf of $F$ and the section $s$ of $F$ is in fact in $H^{0}(G)$ so that $\delta=1$. Similarly, the short exact sequence

$$
0 \rightarrow(0, G) \rightarrow(1, F) \rightarrow(1, F / G) \rightarrow 0
$$

now means that the section $s$ of $F$ does not factor through $G$ and hence $\delta=0$ and we take the image of the section $s$ in $F / G$.

Similarly as in the sheaf case, we can define a Jordan-Hölder filtration and S-equivalence classes. The S-equivalence classes of $\alpha$-semistable pairs form a projective moduli space $\mathrm{M}^{\alpha}(P(m))$ [22]. We say that $\alpha \in \mathbb{Q}$ is a wall if there is a strictly $\alpha$-semistable pairs. As we change $\alpha$, the moduli space $\mathrm{M}^{\alpha}(P(m))$ changes only at walls [32]. A simple reason for this is that if there is no strictly semistable pairs, then $\alpha$-stability does not change by a small perturbation of $\alpha$ because the stability condition is defined by a strict inequality. After we fix the 
Hilbert polynomial $P(m)$, it is easy to see there are only finitely many walls. By the wall crossing at $\alpha_{0}$ we mean to compare the moduli spaces for $\alpha_{-}<\alpha_{0}<\alpha_{+}$ that are sufficiently close to $\alpha_{0}$, that is, there is no other walls between $\alpha_{-}$and $\alpha_{+}$.

What happens at the wall $\alpha_{0}$ is roughly as follows. Let $(1, G) \oplus(0, H)$ be a strictly $\alpha_{0}$-semistable pair, where $(1, G)$ and $(0, H)$ are $\alpha_{0}$-stable pair. Then the pair $(1, F)$ obtained by a nonsplit extension

$$
0 \rightarrow(0, H) \rightarrow(1, F) \rightarrow(1, G) \rightarrow 0
$$

is certainly not $\alpha_{-}$-stable. But in a good situation as in our case, it can be checked that they are all $\alpha_{+}$-stable. Meanwhile, the pair $\left(1, F^{\prime}\right)$ obtained by a nonsplit extension

$$
0 \rightarrow(1, G) \rightarrow\left(1, F^{\prime}\right) \rightarrow(0, H) \rightarrow 0
$$

is certainly not $\alpha_{+}$-stable, but in a good situation it is $\alpha_{-}$-stable. So when crossing the wall, the pairs of the form (1) are replaced by the pairs of the form (2). This can be explained by the elementary modification of the pair. See [5;32] for more details.

By the definition of $\alpha$-stability, as $\alpha$ tends to infinity (for short, $\alpha=\infty$ ), the cokernel of the pair $s: \mathcal{O} \rightarrow F$ is supported on a zero-dimensional scheme (possibly empty). In other words, we get the moduli space of Pandharipande-Thomas stable pairs. On the other hand, when $\alpha$ is sufficiently small (for short $\alpha=0^{+}$), $\alpha$ stability is equivalent to the Gieseker stability of the sheaf. Thus, by wall-crossing, conditions on the section are replaced by conditions on the sheaf. Now, since there is no condition on sections, we get a map to our moduli space $\mathrm{M}(P(m))$ of sheaves by forgetting the section.

When $P(m)=4 m+1$, we see that there is only one wall at $\alpha=3$ ([5]). Strictly $\alpha$-semistable pair in this case is $\left(0, \mathcal{O}_{L}\right) \oplus(1, G)$, where $L$ is a line, and $G$ is a sheaf with Hilbert polynomial $3 m$. The pair given by the extension

$$
0 \rightarrow\left(0, \mathcal{O}_{L}\right) \rightarrow(1, F) \rightarrow(1, G) \rightarrow 0
$$

is unstable for $\alpha<3$ because $\frac{1}{1}>\frac{1+\alpha}{4}$. After crossing the wall, this pair is modified into a pair given by the "flipped" extension

$$
0 \rightarrow(1, G) \rightarrow(1, F) \rightarrow\left(0, \mathcal{O}_{L}\right) \rightarrow 0
$$

The wall crossing picture is as follows.

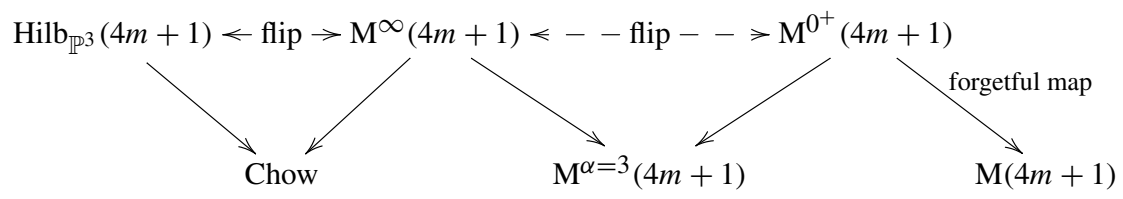

Here the Chow variety is defined by

$$
\text { Chow }=\coprod_{g=0,1,3}\left[C M^{4 m+1-g} \times S^{g}\left(\mathbb{P}^{3}\right)\right]
$$


where $C M^{4 m+1-g}$ is the space of Cohen-Macaulay (shortly, CM) curves with Hilbert polynomial $4 m+1-g$, and $S^{g}\left(\mathbb{P}^{3}\right)$ is the $g$-fold symmetric product. Note that there does not exist any CM curve of genus $g=2$ in $\mathbb{P}^{3}$ ([16, Thm. 3.3]).

\section{LEMMA 2.2.}

(1) If a pair $(s, F)$ is $\alpha$-semistable, then the (scheme theoretic support) of $F$ is a CM-curve.

(2) A pair $(s, F)$ is $\infty$-stable if and only if the cokernel of $s$ is supported on a zero-dimensional subscheme of the support of $F$.

(3) Assume that $\operatorname{gcd}(d, \chi)=1$. Then $(s, F)$ is $0^{+}$-stable if and only if $F$ is a stable sheaf.

Proof. Part (1) is by the purity of $F$. Part (2) directly comes from [28, Section 1.3]. For part (3), when $\operatorname{gcd}(d, \chi)=1$, there is no strictly (Gieseker) semistable sheaves. When $\alpha=0$, the $\alpha$-stability is the same as the Gieseker stability by definition, and a small perturbation of $\alpha$ does not change the stability.

\section{REMARK 2.3.}

(1) By a simple calculation we can see that there are no walls for $d=1,2$, so $\mathrm{M}^{\alpha}(d m+1)$ does not change as $\alpha$ varies.

(2) As a first nontrivial case, where the Hilbert polynomial is $P(m)=3 m+1$, Freiermuth and Trautmann proved that $\mathrm{M}(3 m+1)$ consists of two smooth irreducible components $R \cup E$. Here $R$ parameterizes the structure sheaves of the twisted cubic curves, and $E$ is isomorphic to the universal cubic plane curve. By analyzing the deformation space of each sheaf in $R \cap E$ they showed that $R$ and $E$ meet transversally. This can be explained as follows by using wall crossing. According to Piene and Schlessinger [36], the Hilbert scheme $\operatorname{Hilb}_{\mathbb{P}^{3}}(3 m+1)$ consists of two irreducible components, rational cubic curves and elliptic curves with a point. After wall-crossing, $\mathrm{M}^{\infty}(3 m+1)$ consists of two irreducible components $R \cup E$ as before. Note that the locus of elliptic curves with a free point is excluded after performing the wall-crossing. There is no wall-crossing for pairs, and all sheaves $F$ satisfy $h^{0}(F)=1$, so that the forgetful map is an isomorphism. Thus, we have

$$
\mathrm{M}^{\infty}(3 m+1) \simeq \mathrm{M}^{0^{+}}(3 m+1) \simeq \mathrm{M}(3 m+1) .
$$

\subsection{Geometry of the Hilbert Scheme}

The irreducible components of the Hilbert scheme $\operatorname{Hilb}_{\mathbb{P} 3}(4 m+1)$ have been described in [4].

Proposition 2.4 ([4, Thm. 4.9]). The Hilbert scheme of curves with Hilbert polynomial $4 m+1$ in $\mathbb{P}^{3}$ consists of four irreducible components:

(1) The closure of the locus of the rational quartic curves.

(2) The closure of the locus of the unions of a line and a planar cubic curve. 
(3) The closure of the elliptic quartic curves with one isolated point.

(4) The closure of the planar quartic curves with three isolated points.

The space of elliptic quartic curves in (3) has been studied in [1; 4]. We remark that every connected CM-curve of degree $d=4$ and genus $g=1$ is a ACM-curve and nonplanar ([16, Thm. 3.3 and Prop. 3.5]).

Proposition 2.5.

- ([1, Section 5]) The closure of CM-curves component in $\mathrm{Hilb}_{\mathbb{P}^{3}}(4 m)$ is obtained from the Grassmannian $\operatorname{Gr}(2,10)$ by blowing up twice, where the blow-up loci are degeneracy loci of determinantal varieties.

- ([1, Thm. 5.2]) The possible types (up to projective equivalence) of defining ideals for the connected CM-curve $C$ are:

(1) $I_{C}=\left\langle q_{1}, q_{2}\right\rangle$, where $q_{1}$ and $q_{2}$ are quadratic polynomials.

(2) $I_{C}=\left\langle x y, x z, y q_{1}+z q_{2}\right\rangle$; here $C$ is the union of a planar cubic curve and a line meeting at a point.

(3) $I_{C}=\left\langle x^{2}, x y, x q_{1}+y q_{2}\right\rangle$; here $C$ is the union of a planar conic curve and a double line with genus -2 .

- ([4, Example $2.8(b)])$ The ideal sheaf of the CM-curve $C$ with Hilbert polynomial $4 m$ has the resolution

$$
0 \rightarrow \mathcal{O}(-4) \oplus \mathcal{O}(-3) \rightarrow \mathcal{O}(-3) \oplus \mathcal{O}(-2)^{\oplus 2} \rightarrow I_{C} \rightarrow 0 .
$$

\section{The Moduli Space $\mathbf{M}^{\infty}(4 m+1)$}

In this section we describe all stable pairs in $\mathbf{M}_{\mathbb{P}^{3}}^{\infty}(4 m+1)$. Considering the Hilbert-Chow morphism and the Hilbert scheme described in Section 2.2, we classify all stable pairs according to their supports.

Lemma 3.1. Let $(s, F) \in \mathrm{M}_{\mathbb{P}^{3}}^{\infty}(4 m+1)$ be a stable pair. Then:

(1) $s: \mathcal{O}_{C} \stackrel{\simeq}{\rightarrow} F$ where $C$ is a rational quartic curve or the disjoint union of a line and a planar cubic curve. Note that $\chi\left(\mathcal{O}_{C}(m)\right)=4 m+1$.

(2) $s: \mathcal{O}_{C} \hookrightarrow F$ such that $\operatorname{Coker}(s)=\mathbb{C}_{p}$ for a point $p \in C$. Note that $\chi\left(\mathcal{O}_{C}(m)\right)=4 m$.

(3) $s: \mathcal{O}_{C} \hookrightarrow F$ such that $K=\operatorname{Coker}(s)$ has dimension zero and length 3. Note that $\chi\left(\mathcal{O}_{C}(m)\right)=4 m-2$.

Proof. By stability the cokernel of $s$ must be supported on a zero-dimensional subscheme of the support of the sheaf (Lemma 2.2). By using the classification of CM-curves given at Section 2.2 and comparing Hilbert polynomials we can check that the given list is complete.

REMARK 3.2. In case (1) of Lemma 3.1 the set of rational quartic curves is nothing but the Hilbert scheme of connected CM-curves with Hilbert polynomial $4 m+1$, which is well known to be irreducible (see [27]). We denote this component by $\mathbf{R}^{\infty}$. We denote by $\mathbf{X}^{\infty}$ the component consisting of disjoint unions of a line and 
a planar cubic curve. This component will be dropped from the moduli space after wall-crossing.

In case (3) the sheaf $F$ must be planar. Let $F$ be the pure sheaf fitting into the short exact sequence

$$
0 \rightarrow \mathcal{O}_{C} \rightarrow F \rightarrow K \rightarrow 0
$$

with $l(K)=3$. By the genus-degree formula the curve $C$ has degree 4 and is contained in a plane $H \subset \mathbb{P}^{3}$. Let us consider the natural restriction map

$$
\phi:\left.F \rightarrow F\right|_{H} .
$$

Since $\left.F\right|_{H}$ has the subsheaf $\mathcal{O}_{C}, \mathcal{K e r}(\phi)$ is zero-dimensional or zero. In the first case the purity of $F$ gets contradicted. Thus, $\left.F \simeq F\right|_{H}$, that is, $F$ is planar. Such sheaves form an irreducible component, denoted $\mathbf{P}^{\infty}$, which is isomorphic to the relative moduli space $\mathbf{M}^{\infty}(\mathbb{P} \mathcal{U}, 4 m+1)$, where $\mathcal{U}$ is the universal rank three bundle over $\operatorname{Gr}(3,4)=\left(\mathbb{P}^{3}\right)^{*}$.

In case (2) of Lemma 3.1 the sheaf $F$ is given by the extension

$$
0 \rightarrow \mathcal{O}_{C} \rightarrow F \rightarrow \mathbb{C}_{p} \rightarrow 0,
$$

where $C$ is as in Proposition 2.5. However, unlike in the degree 3 case studied by Freiermuth and Trautmann, the sheaf $F$ may not be uniquely determined by a pair $(p, C)$, that is, the extension group $\operatorname{Ext}^{1}\left(\mathbb{C}_{p}, \mathcal{O}_{C}\right)$ can have dimension greater than one. In fact, $\operatorname{dim} \operatorname{Ext}^{1}\left(\mathbb{C}_{p}, \mathcal{O}_{C}\right) \leq 2$ (see Lemma 3.3). We denote the locus of such pairs by $\mathbf{E}^{\infty}=\mathbf{E}_{1}^{\infty} \cup \mathbf{E}_{2}^{\infty}$, where the lower index is $\operatorname{dim} \operatorname{Ext}^{1}\left(\mathbb{C}_{p}, \mathcal{O}_{C}\right)$.

Lemma 3.3. Let $C$ be a CM-curve with Hilbert polynomial $4 m$, and let $p \in C$. Then

$$
1 \leq \operatorname{dim} \operatorname{Ext}^{1}\left(\mathbb{C}_{p}, \mathcal{O}_{C}\right) \leq 2 .
$$

Moreover, $\operatorname{Ext}^{1}\left(\mathbb{C}_{p}, \mathcal{O}_{C}\right) \simeq \mathbb{C}^{2}$ precisely when (up to projective equivalence)

(1) $I_{C}=\left\langle x y, x z, y q_{1}+z q_{2}\right\rangle$ and $q_{1}(p)=q_{2}(p)=0$,

(2) $I_{C}=\left\langle x^{2}, x y, x q_{1}+y q_{2}\right\rangle$ and $q_{1}(p)=q_{2}(p)=0$.

Proof. By Serre duality ([18, Thm. 3.12]) and using the short exact sequence

$$
0 \rightarrow I_{C} \rightarrow \mathcal{O}_{\mathbb{P}^{3}} \rightarrow \mathcal{O}_{C} \rightarrow 0,
$$

we have the isomorphisms

$$
\operatorname{Ext}^{1}\left(\mathbb{C}_{p}, \mathcal{O}_{C}\right) \simeq \operatorname{Ext}^{2}\left(\mathcal{O}_{C}, \mathbb{C}_{p}\right)^{*} \simeq \operatorname{Ext}^{1}\left(I_{C}, \mathbb{C}_{p}\right)^{*} .
$$

From the resolution of $I_{C}$ at Proposition 2.5 we get the exact sequence

$$
\begin{aligned}
& 0 \rightarrow \operatorname{Ext}^{0}\left(I_{C}, \mathbb{C}_{p}\right) \rightarrow \operatorname{Ext}^{0}\left(\mathcal{O}(-3) \oplus 2 \mathcal{O}(-2), \mathbb{C}_{p}\right) \\
& \stackrel{\delta}{\rightarrow} \operatorname{Ext}^{0}\left(\mathcal{O}(-4) \oplus \mathcal{O}(-3), \mathbb{C}_{p}\right) \rightarrow \operatorname{Ext}^{1}\left(I_{C}, \mathbb{C}_{p}\right) \rightarrow 0 .
\end{aligned}
$$

Now we calculate the rank of $\delta$ for each case described in Proposition 2.5. Without loss of generality, we may assume that $p=[0: 0: 0: 1]$. 
(1) If $C$ is general, meaning $I_{C}=\left\langle q_{1}, q_{2}\right\rangle$, then $\delta$ is given by the matrix

$$
\left[\begin{array}{cc}
0 & -q_{2} \\
0 & q_{1} \\
1 & 0
\end{array}\right],
$$

which has rank 1 at $p$. Thus, $\operatorname{Ext}^{1}\left(\mathbb{C}_{p}, \mathcal{O}_{C}\right) \simeq \mathbb{C}$.

(2) Assume that $C$ is the union of the line $L$ and cubic curve $C_{0}$. Write

$$
I_{C}=\left\langle x y, x z, y q_{1}+z q_{2}\right\rangle, \quad I_{L}=\langle y, z\rangle, \quad I_{C_{0}}=\left\langle x, y q_{1}+z q_{2}\right\rangle .
$$

Then $\delta$ is given by the matrix

$$
\left[\begin{array}{cc}
-q_{1} & z \\
-q_{2} & -y \\
x & 0
\end{array}\right],
$$

whose rank at $p$ depends on the position of $p$ as follows:

(a) $\operatorname{rank}(\delta(p))=0$ if and only if $p \in \operatorname{sing}\left(C_{0}\right)$ (i.e., $q_{1}(p)=q_{2}(p)=0$ ) and $p \in L$. In this case, $\operatorname{Ext}^{1}\left(\mathbb{C}_{p}, \mathcal{O}_{C}\right) \simeq \mathbb{C}^{2}$

(b) $\operatorname{rank}(\delta(p))=1$ otherwise. In this case, $\operatorname{Ext}^{1}\left(\mathbb{C}_{p}, \mathcal{O}_{C}\right) \simeq \mathbb{C}$.

(3) Assume that $C$ is the union of a conic curve and a double line of genus -2 . Write

$$
I_{C}=\left\langle x^{2}, x y, x q_{1}+y q_{2}\right\rangle, \quad I_{L}=\langle x, y\rangle, \quad I_{Q}=\left\langle x, q_{2}\right\rangle .
$$

Then $\delta$ is given by the matrix

$$
\left[\begin{array}{cc}
-q_{1} & -y \\
-q_{2} & x \\
x & 0
\end{array}\right] .
$$

As before, we see that $\operatorname{Ext}^{1}\left(\mathbb{C}_{p}, \mathcal{O}_{C}\right) \simeq \mathbb{C}^{2}$ if and only if $q_{1}(p)=q_{2}(p)=0$. This happens when $p \in L \cap Q$ and $q_{1}(p)=0$.

We denote by $\langle C\rangle$ the maximal linear space containing the curve $C$. From Lemma 3.3 we obtain the following:

Proposition 3.4. The moduli space $\mathrm{M}^{\infty}(4 m+1)$ is the union $\mathbf{R}^{\infty} \cup \mathbf{E}^{\infty} \cup \mathbf{P}^{\infty} \cup$ $\mathbf{X}^{\infty}$. Furthermore, $\mathbf{E}^{\infty}=\mathbf{E}_{1}^{\infty} \cup \mathbf{E}_{2}^{\infty}$, where

(1) $\mathbf{E}_{1}^{\infty}$ is the set of nonsplit extensions of $\mathbb{C}_{p}$ by $\mathcal{O}_{C}$ such that one of the following holds:

(a) $I_{C}=\left\langle q_{1}, q_{2}\right\rangle$;

(b) $I_{C}=I_{L \cup C_{0}}$, where $C_{0}$ is a planar cubic curve, $L$ is a line meeting $C_{0}$ at a point, and $p$ is a point in $C_{0}$ such that either $p \notin \operatorname{sing}\left(C_{0}\right)$ or $p \notin L$;

(c) $I_{C}=\left\langle x^{2}, x y, x q_{1}+y q_{2}\right\rangle$ after a change of coordinates, and $p$ is a point on $C$ such that $p \notin L$ or $q_{1}(p) \neq 0$ or $q_{2}(p) \neq 0$;

(2) $\mathbf{E}_{2}^{\infty}$ is the set of nonsplit extensions of $\mathbb{C}_{p}$ by $\mathcal{O}_{C}$ such that one of the following holds:

(a) $I_{C}=I_{L \cup C_{0}}, L \nsubseteq\left\langle C_{0}\right\rangle,\{p\}=C \cap L \subset \operatorname{sing}\left(C_{0}\right)$ for a line $L$ and a cubic curve $C_{0}$; 
(b) $I_{C}=\left\langle x^{2}, x y, x q_{1}+y q_{2}\right\rangle$ after a change of coordinates, and $p$ is a point on $C$ such that $p \in L$ and $q_{1}(p)=q_{2}(p)=0$.

In the cases (1)(c) and (2)(b) we frequently write $C=L^{2} Q$, where $Q$ is the conic defined by $\left\langle x, q_{2}\right\rangle$, and $L$ is the line $\langle x, y\rangle$, because $C$ is a union of $Q$ and a double line supported on $L$.

LEMma 3.5. Let $E \subset \mathrm{Hilb}_{\mathbb{P}^{3}}(4 m)$ be the locus of connected locally CM-curves. Let $\mathcal{C}=\{(p, C) \mid C \in E, p \in C\}$ be the universal family of $E$. Then $\mathcal{C}$ is an irreducible variety of dimension 17 . Consequently, $\mathbf{E}_{1}^{\infty}$ is an irreducible variety of dimension 17.

Proof. The projection map $\pi: \mathcal{C} \subset E \times \mathbb{P}^{3} \rightarrow E$ is flat. By [1] we know that $E$ is an irreducible variety. Now we apply [15, III. Prop. 9.6]. For each $e \in E$, every irreducible component of the fiber $\pi^{-1}(e)$ has dimension one. Thus, every irreducible component of $\mathcal{C}$ has dimension $16+1=17$. By Proposition $2.5, E$ is a blown-up space of a Grassmannian variety. Thus, the inverse image of $\pi$ away from the exceptional locus in $E$ is irreducible. But the inverse image of the exceptional locus in $E$ has dimension 16, and hence it does not form a new irreducible component of $\mathcal{C}$.

\section{Proof of the Main Theorem}

We use the wall-crossing of $\alpha$-stable pairs to relate $\mathbf{M}^{\infty}(4 m+1)$ with $\mathrm{M}^{0^{+}}(4 m+1)$. Note that since a sheaf in $\mathrm{M}(4 m+1)$ has at least one nonzero section, the forgetful map from $\mathrm{M}^{0^{+}}(4 m+1)$ to $\mathrm{M}(4 m+1)$ is surjective. Moreover, we have the following:

Lemma 4.1. For $F \in \mathrm{M}(4 m+1)$, we have $1 \leq h^{0}(F) \leq 2$. Moreover, if $h^{0}(F)=2$, then $F$ must be planar.

Proof. This is clear from the possible resolutions of $F$ found at Section 6.

When $F$ is nonplanar, by choosing the unique nonzero section, we will regard a sheaf as a pair. The locus of planar sheaves was studied in [5].

We will use the following well-known lemma frequently.

LEMmA 4.2. Let $X$ be a projective scheme, and $Y \subset X$ a closed subscheme. Let $F$ be a coherent $\mathcal{O}_{X}$-module, and let $G$ be a coherent $\mathcal{O}_{Y}$-module. Then there is an exact sequence of vector spaces

$$
\begin{aligned}
0 & \rightarrow \operatorname{Ext}_{\mathcal{O}_{Y}}^{1}\left(F_{\mid Y}, G\right) \rightarrow \operatorname{Ext}_{\mathcal{O}_{X}}^{1}(F, G) \rightarrow \operatorname{Hom}_{\mathcal{O}_{Y}}\left(\mathcal{T}_{o r}{ }_{1}^{\mathcal{O}_{X}}\left(F, \mathcal{O}_{Y}\right), G\right) \\
& \rightarrow \operatorname{Ext}_{\mathcal{O}_{Y}}^{2}\left(F_{\mid Y}, G\right) \rightarrow \operatorname{Ext}_{\mathcal{O}_{X}}^{2}(F, G) .
\end{aligned}
$$

In particular, if $F$ is an $\mathcal{O}_{Y}$-module, then there is an exact sequence

$$
\begin{aligned}
0 & \rightarrow \operatorname{Ext}_{\mathcal{O}_{Y}}^{1}\left(F_{\mid Y}, G\right) \rightarrow \operatorname{Ext}_{\mathcal{O}_{X}}^{1}(F, G) \rightarrow \operatorname{Hom}_{\mathcal{O}_{Y}}\left(F \otimes_{\mathcal{O}_{X}} \mathcal{I}_{Y}, G\right) \\
& \rightarrow \operatorname{Ext}_{\mathcal{O}_{Y}}^{2}\left(F_{\mid Y}, G\right) \rightarrow \operatorname{Ext}_{\mathcal{O}_{X}}^{2}(F, G) .
\end{aligned}
$$


Proof. The results directly come from the existence of the base change spectral sequence ([26, Thm. 12.1]):

$$
\operatorname{Ext}_{\mathcal{O}_{Y}}^{p}\left(\mathcal{T}_{o r}^{\mathcal{O}_{X}}\left(F, \mathcal{O}_{Y}\right), G\right) \Rightarrow \operatorname{Ext}_{\mathcal{O}_{X}}^{p+q}(F, G) .
$$

REMARK 4.3. In view of the lemma, the results in [12, Thm. 1.1] can be read as follows. Using the wall-crossing (Remark 2.3), we can see that $\mathrm{M}(3 m+1)$ consists of two components:

(1) Nonplanar sheaves, that is, the structure sheaves of CM-curves of degree 3;

(2) Planar sheaves.

The dimensions of each component are 12 and 13, respectively. Moreover, it was shown in [12, Thm. 1.1] that the intersection of these two components consists of singular planar sheaves, that is, planar sheaves that are not locally free on their support. The space of CM-curves is quasi-projective and smooth, cf. [36, Section 4]. Let $F$ be a stable sheaf supported on a curve $C$ that is contained in a plane $H \subset \mathbb{P}^{3}$. From (5) we have

$$
\begin{aligned}
0 & \rightarrow \operatorname{Ext}_{H}^{1}(F, F) \rightarrow \operatorname{Ext}_{\mathbb{P}^{3}}^{1}(F, F) \rightarrow \operatorname{Hom}_{H}(F, F(1)) \\
& \rightarrow \operatorname{Ext}_{H}^{2}(F, F)=\operatorname{Ext}_{H}^{0}(F, F(-3))^{*}=0 .
\end{aligned}
$$

By the Grothendieck-Riemann-Roch theorem, $\operatorname{dim}_{\operatorname{Ext}_{H}^{1}}(F, F)=10$. Now, if $F$ is a locally free $\mathcal{O}_{C}$-module, then

$$
\operatorname{Hom}_{H}(F, F(1)) \simeq \mathrm{H}^{0}\left(C, F^{*} \otimes F(1)\right) \simeq \mathrm{H}^{0}\left(\mathcal{O}_{C}(1)\right) \simeq \mathbb{C}^{3} ;
$$

hence, $F$ gives a smooth point in the moduli space $\mathrm{M}(3 m+1)$. If $F$ is not locally free on its support, then from the exact sequence

$$
0 \rightarrow \mathcal{O}_{C} \rightarrow F \rightarrow \mathbb{C}_{p} \rightarrow 0
$$

we deduce that $\operatorname{dim} \operatorname{Hom}(F, F(1)) \leq 4$. According to [12, Thm. 1.1], this is actually an equality, and hence $F$ gives a singular point in $\mathrm{M}(3 m+1)$.

The numerical type of wall of the spaces $\mathrm{M}^{\alpha}(4 m+1)$ is given by the following lemma.

LEMma 4.4. The wall-crossing on $\mathrm{M}^{\alpha}(4 m+1)$ occurs at $\alpha=3$ with the JordanHölder filtration

$$
\left(0, \mathcal{O}_{L}\right) \oplus\left(1, \mathcal{O}_{C_{0}}\right),
$$

where $C_{0}$ is a planar cubic curve.

Proof. By numerical computation the possible type of wall is given by

$$
\left(0, F_{m+1}\right) \oplus\left(1, F_{3 m}\right),
$$

where the subscripts indicate the Hilbert polynomials of the sheaves (Section 2.1). Obviously, $F_{m+1} \simeq \mathcal{O}_{L}$. The sheaf $F_{3 m}$ is planar because it has a section and is 
pure. Thus, $F_{3 m}$ is isomorphic to the structure sheaf of a planar cubic curve ([11, p. 18]).

By wall-crossing the pairs in $\mathrm{M}^{\infty}(4 m+1)$ of the form

$$
0 \rightarrow\left(0, \mathcal{O}_{L}\right) \rightarrow(1, F) \rightarrow\left(1, \mathcal{O}_{C_{0}}\right) \rightarrow 0
$$

are modified into pairs in $\mathrm{M}^{0^{+}}(4 m+1)$ of the form

$$
0 \rightarrow\left(0, \mathcal{O}_{C_{0}}\right) \rightarrow(1, F) \rightarrow\left(1, \mathcal{O}_{L}\right) \rightarrow 0 .
$$

We call the set of such pairs the wall-crossing locus.

The immediate consequence of Lemma 4.4 is that $\mathbf{X}^{\infty}$ is dropped after the wall-crossing. We denote by $\mathbf{R}^{+}, \mathbf{E}_{1}^{+}, \mathbf{E}_{2}^{+}$, and $\mathbf{P}^{+}$the loci in $\mathbf{M}^{0^{+}}(4 m+1)$ corresponding to $\mathbf{R}^{\infty}, \mathbf{E}_{1}^{\infty}, \mathbf{E}_{2}^{\infty}$, and $\mathbf{P}^{\infty}$, respectively. Away from the wall-crossing locus, the moduli space is unchanged. By Lemma 4.1, after forgetting the section, we have $\mathbf{R}^{+} \simeq \mathbf{R}, \mathbf{E}_{1}^{+} \simeq \mathbf{E}_{1}$, and $\mathbf{E}_{2}^{+} \simeq \mathbf{E}_{2}$.

\section{LEMMA 4.5.}

(1) The locus $\mathbf{R}^{\infty}$ does not intersect the wall-crossing locus. Thus, $\mathbf{R}^{\infty} \simeq \mathbf{R}^{+}$.

(2) The locus $\mathbf{P}^{+}$is the relative $0^{+}$-stable pairs space $\mathrm{M}^{0^{+}}(\mathbb{P U}, 4 m+1)$, where $\mathcal{U}$ is the universal rank three bundle over the Grassmannian $\operatorname{Gr}(3,4)=\left(\mathbb{P}^{3}\right)^{*}$. In particular, $\mathbf{P}^{+}$is irreducible.

Proof. The structure sheaf $\mathcal{O}_{C}$ of a connected CM-rational quartic curve $C \in \mathbf{R}^{\infty}$ does not fit into the short exact sequence (6) because of $h^{0}\left(\mathcal{O}_{C}\right)=1$, and hence (1) follows. For (2), we can apply the wall-crossing of [5]. Note that, by the results of [5, Prop. 4.4], $\mathbf{M}_{\mathbb{P}^{2}}^{0^{+}}(4 m+1)$ is a blow-up of $\mathbf{M}_{\mathbb{P}^{2}}(4 m+1)$ along the Brill-Noether locus, and hence it is irreducible. It follows that $\mathbf{P}^{+}$is irreducible.

Thus far, we have shown that $\mathbf{R}^{+}$and $\mathbf{P}^{+}$are irreducible components of $\mathrm{M}^{0^{+}}(4 m+1)$. We denote the nonplanar wall crossing loci by $W^{\infty}$ and $W^{+}$, respectively. We will next show that $\mathrm{M}^{0^{+}}(4 m+1) \backslash\left(\mathbf{R}^{+} \cup \mathbf{P}^{+}\right)$is contained in the closure of $\mathbf{E}_{1}^{+} \backslash W^{+}$, denoted $\mathbf{E}^{+}$, which is irreducible by Lemma 3.5.

Recall that in Proposition 3.4 we decomposed $\mathbf{E}_{2}^{\infty}$ into two subsets according to whether the support of the sheaf is the union of a line with a cubic curve or the union of a double line with a conic curve. After wall-crossing, we also have the two corresponding loci, which we denote $\mathbf{E}_{2 a}^{+}$and $\mathbf{E}_{2 b}^{+}$.

Proposition 4.6. We have the decomposition $\mathbf{E}_{2}^{+} \backslash W^{+}=\mathbf{E}_{2 a}^{+} \cup \mathbf{E}_{2 b}^{+}$, where

(1) $\mathbf{E}_{2 a}^{+}$is the union of sets of the form $\mathbb{P}\left(\operatorname{Ext}^{1}\left(\mathbb{C}_{p}, \mathcal{O}_{C}\right)\right) \backslash \mathbb{P}\left(\operatorname{Ext}^{1}\left(\mathbb{C}_{p}\right.\right.$, $\left.\left.\mathcal{O}_{L}(-1)\right)\right) \simeq \mathbb{P}^{1} \backslash\{\mathrm{pt}\}$, with $C=L \cup C_{0}$ for a cubic curve $C_{0}, L \nsubseteq\left\langle C_{0}\right\rangle$, $\{p\}=C \cap L \subset \operatorname{sing}\left(C_{0}\right)$;

(2) $\mathbf{E}_{2 b}^{+}$is the union of sets of the form $\mathbb{P}\left(\operatorname{Ext}^{1}\left(\mathbb{C}_{p}, \mathcal{O}_{C}\right)\right) \backslash \mathbb{P}\left(\operatorname{Ext}^{1}\left(\mathbb{C}_{p}\right.\right.$, $\left.\left.\mathcal{O}_{L}(-1)\right)\right) \simeq \mathbb{P}^{1} \backslash\{\mathrm{pt}\}$, with $C=L^{2} \cup Q$ for a conic curve $Q, L \subset\langle Q\rangle$, $p \in L, p_{1}(p)=p_{2}(p)=0$. 
Proof. Consider the exact sequence

$$
\begin{aligned}
0 & =\operatorname{Ext}^{0}\left(\mathbb{C}_{p}, \mathcal{O}_{C_{0}}\right) \rightarrow \operatorname{Ext}^{1}\left(\mathbb{C}_{p}, \mathcal{O}_{L}(-1)\right) \simeq \mathbb{C} \\
& \rightarrow \operatorname{Ext}^{1}\left(\mathbb{C}_{p}, \mathcal{O}_{C}\right) \stackrel{\delta}{\rightarrow} \operatorname{Ext}^{1}\left(\mathbb{C}_{p}, \mathcal{O}_{C_{0}}\right) \simeq \mathbb{C} .
\end{aligned}
$$

If $F \in \mathbb{P}\left(\operatorname{Ext}^{1}\left(\mathbb{C}_{p}, \mathcal{O}_{C}\right)\right)$ has the nonzero image $G=\delta(F)$, then $F$ fits into the nonsplit short exact sequence

$$
0 \rightarrow \mathcal{O}_{L}(-1) \rightarrow F \rightarrow G \rightarrow 0 .
$$

By Lemma 4.7, $F$ is a stable sheaf, and, thus, it does not contain $\mathcal{O}_{L}$.

LEMMA 4.7. Let $G$ be a stable sheaf with Hilbert polynomial $(d-1) m+1$. Let $L$ be a line. Then, every sheaf $F$ fitting into the nonsplit short exact sequence

$$
0 \rightarrow \mathcal{O}_{L}(-1) \rightarrow F \rightarrow G \rightarrow 0
$$

is stable with Hilbert polynomial $\mathrm{dm}+1$.

Proof. Let $F^{\prime}$ be a subsheaf of $F$. Let $G^{\prime}$ be its image in $G$, and $K=F^{\prime} \cap$ $\mathcal{O}_{L}(-1)$. We have $\chi(K) \leq 0$. If $G^{\prime} \neq G$, then $\chi\left(G^{\prime}\right) \leq 0$, and hence $\chi\left(F^{\prime}\right) \leq 0$. If $G^{\prime}=G$, then $K \neq 0$ and $K \neq \mathcal{O}_{L}(-1)$, hence $\chi(K) \leq-1$, and hence $\chi\left(F^{\prime}\right) \leq 0$.

Next, we will show that the wall-crossing locus is contained in $\mathbf{E}^{+}$.

LEMma 4.8. The variety $W^{+} \cup \mathbf{E}_{2 a}^{+}$is irreducible of dimension 15 and is contained in $\mathbf{E}^{+}$.

Proof. Consider the extension

$$
0 \rightarrow \mathcal{O}_{C_{0}} \rightarrow F \rightarrow \mathcal{O}_{L} \rightarrow 0 .
$$

Denote $\{p\}=L \cap C_{0}$. Let $H$ be the plane containing $C_{0}$. Tensoring (8) with $\mathcal{O}_{H}$, we get the exact sequence

$$
0=\mathcal{T o r}_{1}^{\mathcal{O}_{\mathbb{P}^{3}}}\left(\mathcal{O}_{L}, \mathcal{O}_{H}\right) \rightarrow \mathcal{O}_{C_{0}} \rightarrow F_{\mid H} \rightarrow \mathbb{C}_{p} \rightarrow 0
$$

from which we see that $F_{\mid H} \simeq \mathcal{O}_{C_{0}}(p)$ because $\operatorname{Ext}_{H}^{1}\left(\mathbb{C}_{p}, \mathcal{O}_{C_{0}}\right)=\mathbb{C}$. We obtain the extension

$$
0 \rightarrow \mathcal{O}_{L}(-1) \rightarrow F \rightarrow \mathcal{O}_{C_{0}}(p) \rightarrow 0 .
$$

Conversely, by Lemma 4.7 any sheaf of this form is stable. Let

$$
T=\left\{\left(p, C_{0}, L\right) \mid\{p\}=L \cap C_{0} \text { and } L \nsubseteq H=\left\langle C_{0}\right\rangle\right\},
$$

where $C_{0}$ is a planar cubic curve contained in the plane $H$. Consider the subset $T_{\text {sing }} \subset T$ given by the condition $p \in \operatorname{sing}\left(C_{0}\right)$. Obviously, $T$ is a $\left(\mathbb{P}^{2} \backslash \mathbb{P}^{1}\right)$-bundle over the universal planar cubic curve. Here $\mathbb{P}^{2} \backslash \mathbb{P}^{1}$ parameterizes the choice of line $L$. The space $T_{\text {sing }}$ has the same bundle structure over the singular cubic curves, and hence $T$ and $T_{\text {sing }}$ are smooth of dimensions 15 and 13, respectively. 
We claim that $W^{+} \cup \mathbf{E}_{2 a}^{+}$is irreducible. Let us consider a $\mathbb{P}^{1}$-bundle over $T$ defined by a relative extension sheaf. Let us denote the natural projection maps:

$$
\begin{aligned}
& q_{1}: T \times \mathbb{P}^{3} \rightarrow \mathbb{P}^{3} \times \mathbb{P}^{3}, \quad q_{2}: T \times \mathbb{P}^{3} \rightarrow \operatorname{Gr}(2,4) \times \mathbb{P}^{3}, \\
& q_{3}: T \times \mathbb{P}^{3} \rightarrow \mathbb{P}\left(\operatorname{Sym}^{3} \mathcal{U}\right) \times \mathbb{P}^{3}, \quad p: T \times \mathbb{P}^{3} \rightarrow T,
\end{aligned}
$$

where $U$ is the universal rank 3-bundle over $\operatorname{Gr}(3,4)=\left(\mathbb{P}^{3}\right)^{*}$. Let $\mathcal{F}_{1}, \mathcal{F}_{2}$, and $\mathcal{F}_{3}$ be the universal families of points, lines, and planar cubics, respectively. Let

$$
\pi: P:=\mathbb{P}\left(\mathcal{E} x t_{p}^{1}\left(q_{1}^{*} \mathcal{F}_{1}, q_{2}^{*} \mathcal{F}_{2}(-1) \oplus q_{3}^{*} \mathcal{F}_{3}\right)\right) \rightarrow T
$$

be the structure morphism of the projectivized relative extension sheaf over $T$. Then the tautological family $\mathcal{F}$ parameterized by $P$ ([19, Example 2.1.12]) defines a rational map

$$
\Psi: P \rightarrow W^{+} \cup \mathbf{E}_{2 a}^{+} \text {. }
$$

By some diagram chasing the map $\Psi$ is well defined on the complement of the union of two disjoint sections $s(T) \cup s^{\prime}(T) \subset P$ consisting of sheaves of the forms $\left\{\mathcal{O}_{L}(-1) \oplus \mathcal{O}_{C_{0}}(p)\right\} \cup\left\{\mathcal{O}_{L} \oplus \mathcal{O}_{C_{0}}\right\}$ because it has the destabilizing quotient sheaf $\mathcal{O}_{L}(-1)$ and $\mathcal{O}_{C_{0}}$, respectively. Because of the existence of relative Quot scheme, it forms a flat family of sheaves $\mathcal{Q}$ over $s(T)$. Let $g: \widetilde{P} \rightarrow P$ be the blowing-up of $P$ along $s(T)$. Let $s \widetilde{(T)}$ be the exceptional divisor. Let

$$
\mathcal{F}^{\prime}:=\operatorname{ker}\left(\left.\widetilde{\mathcal{F}} \rightarrow \widetilde{\mathcal{F}}\right|_{s(T) \times \mathbb{P}^{3}} \rightarrow \widetilde{\mathcal{Q}}\right)
$$

be the composition of the surjective maps, where $\widetilde{F}$ denotes the pull-back of a sheaf $F$ along the map $g \times$ id $: \widetilde{P} \times \mathbb{P}^{3} \rightarrow P \times \mathbb{P}^{3}$. Then the modification has the effect of the change of the subsheaf and quotient sheaf (cf. [5, Def. 2.5]). To finish the proof of our claim, it is enough to show that all stable sheaves parameterized by $W^{+} \cup \mathbf{E}_{2 a}^{+}$aries from this modification. That is, let us show that the normal space

$$
N_{s(T) / P, s} \rightarrow \operatorname{Ext}_{\mathbb{P}^{3}}^{1}\left(\mathcal{O}_{C_{0}}(p), \mathcal{O}_{L}(-1)\right)
$$

is surjective, where $s:=g(v)=\left[\mathcal{O}_{L}(-1) \oplus \mathcal{O}_{C_{0}}(p)\right]$ for $v \in s \widetilde{(T)}$. Note that there is an inclusion $N_{T_{\text {sing }} / T, \pi(s)} \subset N_{s(T) / P, s}$, and the former space is isomorphic to $\operatorname{Ext}_{C_{0}}^{1}\left(\mathbb{C}_{p}, \mathbb{C}_{p}\right)^{*}([20$, Section 3.1.1]). Also,

$$
\begin{aligned}
\operatorname{Ext}_{C_{0}}^{1}\left(\mathbb{C}_{p}, \mathbb{C}_{p}\right)^{*} & \cong \operatorname{Hom}_{C_{0}}\left(I_{p, C_{0}}, \mathbb{C}_{p}\right)^{*} \cong \operatorname{Ext}_{C_{0}}^{1}\left(\mathbb{C}_{p}, I_{p, C_{0}}\right) \cong \operatorname{Ext}_{H}^{1}\left(\mathbb{C}_{p}, I_{p, C_{0}}\right) \\
& \cong \operatorname{Ext}_{\mathbb{P}^{3}}^{1}\left(\mathcal{O}_{L}(-1), I_{p, C_{0}}\right) \cong \operatorname{Ext}_{\mathbb{P}^{3}}^{1}\left(\mathcal{O}_{C_{0}}(p), \mathcal{O}_{L}(-1)\right),
\end{aligned}
$$

where the first one comes from $0 \rightarrow I_{p, C_{0}} \rightarrow \mathcal{O}_{C_{0}} \rightarrow \mathbb{C}_{p} \rightarrow 0$. The second one comes from the fact that the dualizing sheaf $\omega_{C_{0}}$ is a line bundle, and thus we can apply a version of Serre duality. The third and fourth ones come from equation (4) and $\left.\mathcal{O}_{L}(-1)\right|_{H} \cong \mathbb{C}_{p}$. The last one is [23, Thm. 13]. Hence, we proved that there exists a surjective (rational) map

$$
\widetilde{\Psi}: \widetilde{P} \rightarrow W^{+} \cup \mathbf{E}_{2 a}^{+},
$$

which finishes the proof of our claim. Since general points in $W^{+} \cup \mathbf{E}_{2 a}^{+}$are contained in $\mathbf{E}^{+}$and since it is irreducible, $W^{+} \cup \mathbf{E}_{2 a}^{+}$itself is contained in $\mathbf{E}^{+}$. 
It remains to show that $\mathbf{E}_{2 b}^{\infty} \backslash W^{\infty}=\mathbf{E}_{2 b}^{+} \backslash W^{+}$is contained in $\mathbf{E}^{+}$. To this end, let $Z$ be the locus of the extension sheaves $F$ fitting into the short exact sequence

$$
0 \rightarrow \mathcal{O}_{L}(-1) \rightarrow F \rightarrow \mathcal{O}_{L Q}(p) \rightarrow 0
$$

such that $p \in Q$. Clearly, $\mathbf{E}_{2 b}^{+}$is contained in $Z$. We will show that $Z$ is irreducible. Since general elements in $Z$ are contained in $\mathbf{E}^{+}$, this proves our assertion. Consider the map

$$
\phi: Z \rightarrow \operatorname{Hilb}_{\mathbb{P}^{2}}(m+1) \times \mathrm{M}_{\mathbb{P}^{2}}(3 m+1), \quad[F] \mapsto\left(L,\left[\mathcal{O}_{L Q}(p)\right]\right) .
$$

It will turn out that $\phi$ is a projective bundle over some irreducible variety. This will prove that $Z$ is an irreducible variety.

Lemma 4.9. The locally closed subset $\mathbf{S} \subset \operatorname{Hilb}_{\mathbb{P}^{2}}(m+1) \times \mathrm{M}_{\mathbb{P}^{2}}(3 m+1)$ of pairs $\left(L,\left[\mathcal{O}_{C_{0}}(p)\right]\right)$ for which $C_{0}=L \cup Q$ and $p \in Q$ for a conic curve $Q \subset \mathbb{P}^{2}$ is irreducible.

Proof. Let $\mathrm{M}_{\mathbb{P}^{2}}^{\infty}(2 m+2)$ be the moduli space of pairs with Hilbert polynomial $2 m+2$. We can easily see that it is isomorphic to the universal conic curve, which is a $\mathbb{P}^{4}$-bundle over $\mathbb{P}^{2}$, so is irreducible ([5, Lemma 2.3]). The morphism

$$
\operatorname{Hilb}_{\mathbb{P}^{2}}(m+1) \times \mathrm{M}_{\mathbb{P}^{2}}^{\infty}(2 m+2) \rightarrow \mathbf{S}, \quad(L, p, Q) \mapsto\left(L, \mathcal{O}_{Q \cup L}(p)\right)
$$

is well defined and surjective. Thus, $\mathbf{S}$ is irreducible.

Proposition 4.10. The locus $Z$ is irreducible of dimension 14.

Proof. In view of Lemma 4.9, it is enough to show that the morphism $Z \rightarrow \mathbf{S}$ is surjective and that its fibers are irreducible of the same dimension. We will prove that

$$
\operatorname{Ext}_{\mathcal{O}_{\mathbb{P}^{3}}}^{1}\left(\mathcal{O}_{C_{0}}(p), \mathcal{O}_{L}(-1)\right) \simeq \mathbb{C}^{4}
$$

for all $\left(L,\left[\mathcal{O}_{C_{0}}(p)\right]\right) \in \mathbf{S}$. From (4) we have the exact sequence

$$
\begin{aligned}
0 & \rightarrow \operatorname{Ext}_{\mathcal{O}_{L}}^{1}\left(\mathcal{O}_{C_{0}}(p)_{\mid L}, \mathcal{O}_{L}(-1)\right) \rightarrow \operatorname{Ext}_{\mathcal{O}^{3}}^{1}\left(\mathcal{O}_{C_{0}}(p), \mathcal{O}_{L}(-1)\right) \\
& \rightarrow \operatorname{Hom}\left(\mathcal{T o r}_{1}^{\mathcal{O}^{3}}\left(\mathcal{O}_{C_{0}}(p), \mathcal{O}_{L}\right), \mathcal{O}_{L}(-1)\right) \rightarrow \operatorname{Ext}_{\mathcal{O}_{L}}^{2}\left(\mathcal{O}_{C_{0}}(p)_{\mid L}, \mathcal{O}_{L}(-1)\right)=0 .
\end{aligned}
$$

Assume firstly that $p \notin L$. The long exact sequence of torsion sheaves associated with the short exact sequence

$$
0 \rightarrow \mathcal{O}_{C_{0}} \rightarrow \mathcal{O}_{C_{0}}(p) \rightarrow \mathbb{C}_{p} \rightarrow 0
$$

yields the isomorphisms

$$
\begin{aligned}
\mathcal{O}_{C_{0}}(p)_{\mid L} & \simeq \mathcal{O}_{C_{0 \mid L}} \simeq \mathcal{O}_{L}, \\
\mathcal{T o r}_{1}{ }^{\mathcal{O}_{\mathbb{P}^{3}}}\left(\mathcal{O}_{C_{0}}(p), \mathcal{O}_{L}\right) & \simeq \mathcal{T}_{o r_{1}}{ }^{\mathcal{O}^{3}}\left(\mathcal{O}_{C_{0}}, \mathcal{O}_{L}\right) \simeq \mathcal{O}_{L}(-1) \oplus \mathcal{O}_{L}(-3) .
\end{aligned}
$$

We obtain the isomorphisms

$$
\begin{aligned}
\operatorname{Ext}_{\mathcal{O}_{L}}^{1}\left(\mathcal{O}_{C_{0}}(p)_{\mid L}, \mathcal{O}_{L}(-1)\right) & =0, \\
\operatorname{Hom}\left(\mathcal{T o r}_{1}{ }^{\mathcal{O}^{3} 3}\left(\mathcal{O}_{C_{0}}(p), \mathcal{O}_{L}\right), \mathcal{O}_{L}(-1)\right) & \simeq \mathbb{C}^{4}
\end{aligned}
$$


Assume now that $p \in L$. We have the exact sequence

$$
\begin{aligned}
0 & \rightarrow 2 \mathcal{O}(-3) \stackrel{\delta}{\rightarrow} 3 \mathcal{O}(-2) \oplus \mathcal{O}(-1) \stackrel{\gamma}{\rightarrow} \mathcal{O}(-1) \oplus \mathcal{O} \rightarrow \mathcal{O}_{C_{0}}(p) \rightarrow 0, \\
\delta & =\left[\begin{array}{cc}
x & 0 \\
0 & x \\
-y & -z \\
0 & -q_{2}
\end{array}\right], \quad \gamma=\left[\begin{array}{cccc}
y & z & x & 0 \\
0 & q_{2} & 0 & x
\end{array}\right],
\end{aligned}
$$

where $p$ is given by the ideal $\langle x, y, z\rangle, L$ is given by the ideal $\langle x, y\rangle$, and $Q$ is given by the ideal $\left\langle x, q_{2}\right\rangle$ ([12, Prop. 3.5]). Tensoring with $\mathcal{O}_{L}$ shows that $\mathcal{O}_{C}(p)_{\mid L}$ is isomorphic to the cokernel of the morphism

$$
\mathcal{O}_{L}(-2) \stackrel{\left[\begin{array}{c}
z_{\mid L} \\
q_{2 \mid L}
\end{array}\right]}{\longrightarrow} \mathcal{O}_{L}(-1) \oplus \mathcal{O}_{L}
$$

and that $\operatorname{Tor}_{1} \mathcal{O}_{\mathbb{P} 3}\left(\mathcal{O}_{C_{0}}(p), \mathcal{O}_{L}\right)$ is isomorphic to the middle cohomology of the sequence

$$
\begin{aligned}
2 \mathcal{O}_{L}(-3) & \stackrel{\delta_{L}}{\rightarrow} 3 \mathcal{O}_{L}(-2) \oplus \mathcal{O}_{L}(-1) \stackrel{\gamma_{L}}{\longrightarrow} \mathcal{O}_{L}(-1) \oplus \mathcal{O}_{L}, \\
\delta_{L}=\left[\begin{array}{cc}
0 & 0 \\
0 & 0 \\
0 & -z_{\mid L} \\
0 & -q_{2 \mid L}
\end{array}\right], \quad \gamma_{L}=\left[\begin{array}{cccc}
0 & z_{\mid L} & 0 & 0 \\
0 & q_{2 \mid L} & 0 & 0
\end{array}\right], &
\end{aligned}
$$

which is isomorphic to the cokernel of the morphism

$$
\mathcal{O}_{L}(-3) \stackrel{\left[\begin{array}{c}
0 \\
z_{\mid L} \\
q_{2 \mid L}
\end{array}\right]}{\longrightarrow} 2 \mathcal{O}_{L}(-2) \oplus \mathcal{O}_{L}(-1) .
$$

By hypothesis, $p$ is a point on $Q$, and hence $z_{\mid L}$ divides $q_{2 \mid L}$. It now becomes clear that

$\mathcal{O}_{C_{0}}(p)_{\mid L} \simeq \mathbb{C}_{p} \oplus \mathcal{O}_{L}$ and $\mathcal{T o r}_{1}^{\mathcal{O}_{\mathbb{P}^{3}}}\left(\mathcal{O}_{C_{0}}(p), \mathcal{O}_{L}\right) \simeq \mathbb{C}_{p} \oplus \mathcal{O}_{L}(-2) \oplus \mathcal{O}_{L}(-1)$.

We obtain the isomorphisms

$$
\begin{aligned}
\operatorname{Ext}_{\mathcal{O}_{L}}^{1}\left(\mathcal{O}_{C_{0}}(p)_{\mid L}, \mathcal{O}_{L}(-1)\right) & \simeq \mathbb{C}, \\
\operatorname{Hom}\left(\mathcal{T o r}_{1}^{\mathcal{O}^{3}}\left(\mathcal{O}_{C_{0}}(p), \mathcal{O}_{L}\right), \mathcal{O}_{L}(-1)\right) & \simeq \mathbb{C}^{3}
\end{aligned}
$$

Summarizing the results obtained thus far, we have the following:

Proposition 4.11. The space $\mathrm{M}^{0^{+}}(4 m+1)$ consists of three irreducible components $\mathbf{R}^{+}, \mathbf{E}^{+}$, and $\mathbf{P}^{+}$.

By forgetting the section, we arrive at our main theorem.

THEOREM 4.12. The moduli space $\mathrm{M}_{\mathbb{P}^{3}}(4 m+1)$ consists of three irreducible components $\mathbf{R}, \mathbf{E}$, and $\mathbf{P}$, whose dimensions are 16,17 , and 20 , respectively. 


\section{The Intersections of the Irreducible Components}

In this section, we describe the intersections $\mathbf{R} \cap \mathbf{P}$ and $\mathbf{E} \cap \mathbf{P}$, and we give a nonexhaustive list of sheaves in $\mathbf{R} \cap \mathbf{E}$.

\subsection{The Intersections $\mathbf{R} \cap \mathbf{P}$ and $\mathbf{E} \cap \mathbf{P}$}

We begin by collecting some known facts about the relation of the space of finite maps to the component $\mathbf{R}$. When a finite map is birational to its image, we call it a birational finite map. By the following proposition the birational finite maps give sheaves in the boundary of the locus of structure sheaves of smooth rational quartic curves.

Proposition 5.1. Let $F_{0}\left(\mathbb{P}^{r}, d\right)$ be the space of finite maps from a genus 0 curve of degree $d$ to $\mathbb{P}^{r}$. Then:

(1) The space $F_{0}\left(\mathbb{P}^{r}, d\right)$ is an irreducible projective variety of dimension $(d+$ 1) $(r+1)-3$.

(2) Consider the rational map

$$
\Psi: F_{0}\left(\mathbb{P}^{r}, d\right) \rightarrow M_{\mathbb{P}^{r}}(d m+1), \quad\left[f: C \rightarrow \mathbb{P}^{r}\right] \mapsto f_{*} \mathcal{O}_{C} .
$$

Then $\Psi$ is injective over the locus of birational finite maps.

Proof. By [14, Thm. 2] the moduli space $\bar{M}_{0}\left(\mathbb{P}^{r}, d\right)$ of stable maps of genus 0 and degree $d$ is an irreducible variety. Also, there exists a small contraction morphism $\bar{M}_{0}\left(\mathbb{P}^{r}, d\right) \rightarrow F_{0}\left(\mathbb{P}^{r}, d\right)$ ([3, Prop. 3.11]). This proves the first claim. Part (2) follows directly from the proof of [3, Prop. 3.18].

Proposition 5.2. Let $H \subset \mathbb{P}^{3}$ be a plane, and let $C \subset H$ be an irreducible quartic curve having three distinct nodal singular points $P_{1}, P_{2}, P_{3}$. Then the unique extension of $\mathbb{C}_{P_{1}} \oplus \mathbb{C}_{P_{2}} \oplus \mathbb{C}_{P_{3}}$ by $\mathcal{O}_{C}$, denoted $\mathcal{O}_{C}\left(P_{1}+P_{2}+P_{3}\right)$, gives a point in $\mathbf{R} \cap \mathbf{P}$. We denote by $(\mathbf{R} \cap \mathbf{P})_{0}$ the set of such sheaves. The intersection $\mathbf{R} \cap \mathbf{P}$ is irreducible and is the closure of $(\mathbf{R} \cap \mathbf{P})_{0}$.

Proof. Let $v: \mathbb{P}^{1} \rightarrow C$ be the normalization map. Since the degree $\operatorname{deg}(v)=4, v$ is an element of the moduli space $F_{0}\left(\mathbb{P}^{3}, 4\right)$ of finite maps of degree 4 . By Proposition $5.1, F_{0}\left(\mathbb{P}^{3}, 4\right)$ is an irreducible variety, and the locus $\operatorname{Map}^{s m}\left(\mathbb{P}^{1}, \mathbb{P}^{3}\right) / \operatorname{PGL}(2)$ of finite maps whose images are smooth is a Zariski dense open subset. We can choose a one-parameter family of finite maps $i_{t}: C_{t} \rightarrow \mathbb{P}^{3}$ such that the general fibers are smooth rational quartic curves and $\lim _{t \rightarrow 0} \mathcal{O}_{C_{t}}=v_{*} \mathcal{O}_{\mathbb{P}^{1}}$. But by part (2) of Proposition 5.1 we know that the direct image sheaf $v_{*} \mathcal{O}_{\mathbb{P}^{1}}$ is stable. This is exactly the unique stable extension given by the normalization sequence

$$
0 \rightarrow \mathcal{O}_{C} \rightarrow v_{*} \mathcal{O}_{\mathbb{P}^{1}} \rightarrow \mathbb{C}_{P_{1}} \oplus \mathbb{C}_{P_{2}} \oplus \mathbb{C}_{P_{3}} \rightarrow 0 \text {. }
$$

We deduce that $\mathcal{O}_{C}\left(P_{1}+P_{2}+P_{3}\right)$ lies in $\mathbf{R} \cap \mathbf{P}$.

Assume that $F$ gives a point in $\mathbf{R} \cap \mathbf{P}$, where $\operatorname{supp}(F) \subset H$. We have $F=$ $\lim _{t \rightarrow 0} \mathcal{O}_{C_{t}}$ for smooth rational quartic curves $C_{t}, t \neq 0$. Let us choose a projection center $O \in \mathbb{P}^{3}$ that does not lie on $H$ or on any of the curves $C_{t}$. Let 
$\pi: \mathbb{P}^{3} \backslash\{O\} \rightarrow H$ be the projection with center $O$. Then $\pi_{*} \mathcal{O}_{C_{t}}$ are stable sheaves for $t \neq 0$ by the argument of the previous paragraph. Also, $\pi_{*} F=F$ because $\operatorname{supp}(F) \subset H$. Thus, $\lim _{t \rightarrow 0}\left(\pi_{*} \mathcal{O}_{C_{t}}\right)=\pi_{*}\left(\lim _{t \rightarrow 0} \mathcal{O}_{C_{t}}\right)=F$. Since $\pi_{*} \mathcal{O}_{C_{t}} \in(\mathbf{R} \cap \mathbf{P})_{0}, F$ is an element of the closure of $(\mathbf{R} \cap \mathbf{P})_{0}$. Obviously, the open part $(\mathbf{R} \cap \mathbf{P})_{0}$ of the intersection has a fibration structure over the Grassmannian $\operatorname{Gr}(3,4)=\left(\mathbb{P}^{3}\right)^{*}$. The fibers are birational with the irreducible variety $F_{0}\left(\mathbb{P}^{2}, 4\right)$ of finite maps. Thus, $(\mathbf{R} \cap \mathbf{P})_{0}$ is irreducible.

Proposition 5.3. For any $F \in \mathbf{R}$, we have $h^{0}(F)=1$.

Proof. For any plane $H \subset \mathbb{P}^{3}$ and any sheaf $F$ giving a point in $\mathrm{M}_{H}(4 m+1)$, the exact sequence (5) reads

$$
\begin{aligned}
0 & \rightarrow \operatorname{Ext}_{\mathcal{O}_{H}}^{1}(F, F) \simeq \mathbb{C}^{17} \rightarrow \operatorname{Ext}_{\mathcal{O}^{3}}^{1}(F, F) \rightarrow \operatorname{Hom}(F(-1), F) \\
& \rightarrow \operatorname{Ext}_{\mathcal{O}_{H}}^{2}(F, F) \simeq \operatorname{Hom}(F, F(-3))^{*}=0 .
\end{aligned}
$$

Assume now that $F$ gives a generic point in $\mathbf{R} \cap \mathbf{P}$ of the form $\mathcal{O}_{C}\left(P_{1}+P_{2}+\right.$ $P_{3}$ ), where $C$ is an irreducible planar quartic curve having distinct nodes at $P_{1}$, $P_{2}, P_{3}$. Then $\operatorname{Hom}(F(-1), F) \simeq \mathbb{C}^{5}$. This shows that $\operatorname{ext}^{1}(F, F) \geq 22$. Since the embedding dimension is upper semicontinuous and $\mathbf{R} \cap \mathbf{P}$ is irreducible, this estimate holds for all sheaves in $\mathbf{R} \cap \mathbf{P}$.

Suppose now that $h^{0}(F)=2$. Then we have $F \simeq \mathcal{O}_{C}(-p)(1)$ for some planar quartic curve $C$ and a point $p \in C$ ([11, Prop. 3.3.4]). Let $H$ be the plane containing $C$. From the exact sequence

$$
0 \rightarrow \mathcal{O}_{C}(-p) \rightarrow \mathcal{O}_{C} \rightarrow \mathbb{C}_{p} \rightarrow 0
$$

we get the exact sequence

$$
\begin{aligned}
0 & =\operatorname{Hom}\left(\mathbb{C}_{p}, F\right) \rightarrow \operatorname{Hom}\left(\mathcal{O}_{C}, F\right) \simeq \mathbb{C}^{2} \rightarrow \operatorname{Hom}\left(\mathcal{O}_{C}(-p), F\right) \\
& \rightarrow \operatorname{Ext}_{\mathcal{O}_{H}}^{1}\left(\mathbb{C}_{p}, F\right) \simeq \operatorname{Ext}_{\mathcal{O}_{H}}^{1}\left(F, \mathbb{C}_{p}\right)^{*} .
\end{aligned}
$$

From the resolution

$$
0 \rightarrow \mathcal{O}_{H}(-3) \oplus \mathcal{O}_{H}(-1) \rightarrow 2 \mathcal{O}_{H} \rightarrow F \rightarrow 0
$$

we see that $\operatorname{Ext}_{\mathcal{O}_{H}}^{1}\left(F, \mathbb{C}_{p}\right)^{*}$ is isomorphic to $\mathbb{C}$ or $\mathbb{C}^{2}$, depending on whether $p$ is a regular or a singular point of $C$.

Thus, $\operatorname{hom}(F(-1), F) \leq 4$. In view of the exact sequence at the beginning of the proof, we deduce that $\operatorname{ext}^{1}(F, F) \leq 21$, from which it follows that $F \notin$ $\mathbf{R} \cap \mathbf{P}$.

Remark 5.4. More generally, let $R_{d}\left(\mathbb{P}^{r}\right)$ be the space of irreducible rational curves in $\mathbb{P}^{r}$ of degree $d$. By using elementary modifications of sheaves ([19, Thm. 2.B.1]) we can easily see that every stable sheaf $F \in \overline{R_{d}\left(\mathbb{P}^{r}\right)} \subset \mathrm{M}_{\mathbb{P}^{r}}(d m+$ 1) satisfies $h^{0}(F)=1$.

Similar arguments as in Proposition 5.2 yield the following: 
Proposition 5.5. Let $H \subset \mathbb{P}^{3}$ be a plane, and let $C \subset H$ be an irreducible quartic curve having two distinct nodal singular points $P_{1}$ and $P_{2}$ and no other singularities. Let $P$ be a regular point of $C$. Then $\mathcal{O}_{C}\left(P_{1}+P_{2}+P\right)$ gives a point in $\mathbf{E} \cap \mathbf{P}$. We denote by $(\mathbf{E} \cap \mathbf{P})_{0}$ the set of such sheaves. The intersection $\mathbf{E} \cap \mathbf{P}$ is irreducible and is the closure of $(\mathbf{E} \cap \mathbf{P})_{0}$.

Remark 5.6. Since $\mathbf{R} \cap \mathbf{P} \subset \mathbf{E} \cap \mathbf{P}$, it follows that $\mathbf{R} \cap \mathbf{P} \subset \mathbf{R} \cap \mathbf{E}$.

\subsection{The Intersection $\mathbf{R} \cap \mathbf{E}$}

The intersection $\mathbf{R} \cap \mathbf{E}$ is difficult to describe. We further provide a list of general sheaves in $\mathbf{R} \cap \mathbf{E}$. By Proposition 5.1 all sheaves in $\mathbf{R}$ are flat limits of sheaves of the form $f_{*} \mathcal{O}_{\tilde{C}}$ for finite maps $f$. When $f$ is not birational, the flat limit can be described by the elementary modification technique, which we will repeatedly use in this section. For the general setting of modification of sheaves, see [19, Thm. 2.B.1]. For the version concerning maps, see [9; 10].

Our first order of business is to show that $W^{+}$is disjoint from $\mathbf{R} \cap \mathbf{E}$. Recall that $W^{+}$is the locus of nonplanar sheaves $F$ that fit into an exact sequence

$$
0 \rightarrow \mathcal{O}_{C_{0}} \rightarrow F \rightarrow \mathcal{O}_{L} \rightarrow 0
$$

for a planar cubic curve $C_{0}$ and an incident line $L$ that is not coplanar with $C_{0}$.

Proposition 5.7. Assume that $F$ gives a point in $W^{+}$. Then $\operatorname{ext}^{1}(F, F)=17$, so $F$ is a smooth point of $\mathbf{E}$.

Proof. From (9) we have the exact sequence

$$
\operatorname{Ext}^{1}\left(F, \mathcal{O}_{L}(-1)\right) \rightarrow \operatorname{Ext}^{1}(F, F) \rightarrow \operatorname{Ext}^{1}\left(F, \mathcal{O}_{C_{0}}(p)\right) .
$$

Denote $\{p\}=L \cap C_{0}$. From (4) we have the exact sequence

$$
\begin{aligned}
0 & \rightarrow \operatorname{Ext}_{\mathcal{O}_{L}}^{1}\left(F_{\mid L}, \mathcal{O}_{L}(-1)\right) \rightarrow \operatorname{Ext}_{\mathcal{O}_{\mathbb{P}^{3}}}^{1}\left(F, \mathcal{O}_{L}(-1)\right) \\
& \rightarrow \operatorname{Hom}\left(\mathcal{T o r}_{1}{ }_{\mathbb{P}^{3}}\left(F, \mathcal{O}_{L}\right), \mathcal{O}_{L}(-1)\right) \rightarrow \operatorname{Ext}_{\mathcal{O}_{L}}^{2}\left(F_{\mid L}, \mathcal{O}_{L}(-1)\right)=0 .
\end{aligned}
$$

From (8) and (9) we have the exact sequences

$$
\mathbb{C}_{p}=\mathcal{O}_{C_{0 \mid L}} \rightarrow F_{\mid L} \rightarrow \mathcal{O}_{L} \rightarrow 0 \quad \text { and } \quad 0 \rightarrow \mathcal{O}_{L}(-1) \rightarrow F_{\mid L} \rightarrow \mathcal{O}_{C_{0}}(p)_{\mid L} \rightarrow 0 .
$$

Note that $\mathcal{O}_{C_{0}}(p)_{\mid L}$ is isomorphic to $\mathbb{C}_{p}$ if $p \in \operatorname{reg}\left(C_{0}\right)$, respectively, to $\mathbb{C}_{p} \oplus \mathbb{C}_{p}$ if $p \in \operatorname{sing}\left(C_{0}\right)$. It follows that $F_{\mid L}$ is isomorphic to $\mathcal{O}_{L}$ if $p \in \operatorname{reg}\left(C_{0}\right)$, respectively, to $\mathbb{C}_{p} \oplus \mathcal{O}_{L}$ if $p \in \operatorname{sing}\left(C_{0}\right)$. From (8) we get the long exact sequence of torsion sheaves of $\mathcal{O}_{\mathbb{P}^{3}}$-modules

$$
\begin{aligned}
\mathbb{C}_{p} & \simeq \mathcal{T o r}_{1}\left(\mathcal{O}_{C_{0}}, \mathcal{O}_{L}\right) \rightarrow \mathcal{T o r}_{1}\left(F, \mathcal{O}_{L}\right) \rightarrow \mathcal{T o r}_{1}\left(\mathcal{O}_{L}, \mathcal{O}_{L}\right) \\
& \simeq 2 \mathcal{O}_{L}(-1) \rightarrow \mathbb{C}_{p} \rightarrow F_{\mid L} \rightarrow \mathcal{O}_{L} \rightarrow 0
\end{aligned}
$$

From this we see that

$$
\operatorname{Tor}_{1}\left(F, \mathcal{O}_{L}\right) / \mathcal{T}^{0}\left(\mathcal{T o r}_{1}\left(F, \mathcal{O}_{L}\right)\right) \simeq \begin{cases}\mathcal{O}_{L}(-2) \oplus \mathcal{O}_{L}(-1) & \text { if } p \in \operatorname{reg}(C) \\ \mathcal{O}_{L}(-1) & \text { if } p \in \operatorname{sing}(C)\end{cases}
$$


In the first case we have the exact sequence

$$
\begin{aligned}
0 & =\operatorname{Ext}_{\mathcal{O}_{L}}^{1}\left(F_{\mid L}, \mathcal{O}_{L}(-1)\right) \rightarrow \operatorname{Ext}_{\mathcal{O}_{\mathbb{P}^{3}}}^{1}\left(F, \mathcal{O}_{L}(-1)\right) \\
& \rightarrow \operatorname{Hom}\left(\mathcal{O}_{L}(-2) \oplus \mathcal{O}_{L}(-1), \mathcal{O}_{L}(-1)\right) \simeq \mathbb{C}^{3} \rightarrow 0 .
\end{aligned}
$$

In the second case we have the exact sequence

$$
\begin{aligned}
0 & \rightarrow \operatorname{Ext}_{\mathcal{O}_{L}}^{1}\left(F_{\mid L}, \mathcal{O}_{L}(-1)\right) \simeq \mathbb{C} \rightarrow \operatorname{Ext}_{\mathcal{O}_{\mathbb{P}^{3}}}^{1}\left(F, \mathcal{O}_{L}(-1)\right) \\
& \rightarrow \operatorname{Hom}\left(2 \mathcal{O}_{L}(-1), \mathcal{O}_{L}(-1)\right) \simeq \mathbb{C}^{2} \rightarrow 0 .
\end{aligned}
$$

In both cases we get the isomorphism $\operatorname{Ext}_{\mathcal{O}_{\mathbb{P} 3}}^{1}\left(F, \mathcal{O}_{L}(-1)\right) \simeq \mathbb{C}^{3}$.

Let $H$ be the plane containing $C_{0}$. From (4), taking into account that $F_{\mid H} \simeq$ $\mathcal{O}_{C_{0}}(p)$, we get the exact sequence

$$
\begin{aligned}
0 & \rightarrow \operatorname{Ext}_{\mathcal{O}_{H}}^{1}\left(F_{\mid H}, \mathcal{O}_{C_{0}}(p)\right) \simeq \mathbb{C}^{10} \rightarrow \operatorname{Ext}_{\mathcal{O}_{\mathbb{P}^{3}}}^{1}\left(F, \mathcal{O}_{C_{0}}(p)\right) \\
& \rightarrow \operatorname{Hom}\left(\mathcal{T o r}_{1}^{\mathcal{O}_{\mathbb{P}^{3}}}\left(F, \mathcal{O}_{H}\right), \mathcal{O}_{C_{0}}(p)\right) \rightarrow \operatorname{Ext}_{\mathcal{O}_{H}}^{2}\left(F_{\mid H}, \mathcal{O}_{C_{0}}(P)\right)=0 .
\end{aligned}
$$

The long exact sequence of torsion sheaves associated to (8) reads in part

$$
\begin{aligned}
0 & =\mathcal{T}_{\operatorname{or}_{2}}\left(\mathcal{O}_{L}, \mathcal{O}_{H}\right) \rightarrow \mathcal{T o r}_{1}\left(\mathcal{O}_{C_{0}}, \mathcal{O}_{H}\right) \simeq \mathcal{O}_{C_{0}}(-1) \\
& \rightarrow \mathcal{T}_{\operatorname{or}_{1}}\left(F, \mathcal{O}_{H}\right) \rightarrow \mathcal{T o r}_{1}\left(\mathcal{O}_{L}, \mathcal{O}_{H}\right)=0 .
\end{aligned}
$$

We obtain the exact sequence

$$
0 \rightarrow \mathbb{C}^{10} \rightarrow \operatorname{Ext}_{\mathcal{O}_{\mathbb{P}^{3}}}^{1}\left(F, \mathcal{O}_{C_{0}}(p)\right) \rightarrow \operatorname{Hom}\left(\mathcal{O}_{C_{0}}(-1), \mathcal{O}_{C_{0}}(p)\right) \rightarrow 0
$$

Thus, $\operatorname{Ext}_{\mathcal{O}_{\mathbb{P}^{3}}}^{1}\left(F, \mathcal{O}_{C_{0}}(p)\right) \simeq \mathbb{C}^{14}$. From the exact sequence at the beginning of the proof we get the inequality $\operatorname{ext}^{1}(F, F) \leq 17$. This must be an equality because the moduli space has dimension 17 at $F$ ([19, Cor. 4.5.2]).

In view of Proposition 5.1, whenever the reduced support of a sheaf $F \in \overline{\mathbf{R}}$ is of degree $4, F \simeq f_{*} \mathcal{O}_{\tilde{C}}$ for some birational finite map $f: \tilde{C} \rightarrow \mathbb{P}^{3}$. The list of such sheaves is as follows.

Proposition 5.8. Let $F \in \mathbf{R} \cap \mathbf{E}$ have reduced support of degree 4. Then one of following holds:

(1) $F$ is the unique nonsplit extension of $\mathbb{C}_{p}$ by $\mathcal{O}_{C}$, where either

(a) $C$ has an ideal $\left\langle q_{1}, q_{2}\right\rangle$ generated by two quadratic polynomials and $p$ is a singular point of $C$; or

(b) $C$ is the union of a planar cubic $C_{0}$ that is singular at $p$ and a line $L$ meeting $C_{0}$ at a regular point.

(2) When $C$ is the union of a planar cubic $C_{0}$ that has a node at $p$ and a line $L$ passing through $p$, only two sheaves in the extension $\mathbb{P}\left(\operatorname{Ext}^{1}\left(\mathbb{C}_{p}, \mathcal{O}_{C}\right)\right) \simeq \mathbb{P}^{1}$ belong to $\mathbf{R} \cap \mathbf{E}$.

(3) When $C$ is the union of a planar cubic $C_{0}$ that has a cusp at $p$ and a line $L$ passing though $p$, precisely one sheaf in the extension $\mathbb{P}\left(\operatorname{Ext}^{1}\left(\mathbb{C}_{p}, \mathcal{O}_{C}\right)\right) \simeq \mathbb{P}^{1}$ belongs to $\mathbf{R} \cap \mathbf{E}$.

The sheaves from (3) lie in the closure of the set of sheaves from (2). 
Proof. Let $C$ be the reduced support of $F$. From Proposition 2.5 we see that either $I_{C}=\left\langle q_{1}, q_{2}\right\rangle$ or $I_{C}=\left\langle x y, x z, y q_{1}+z q_{2}\right\rangle$ after a change of coordinates, where $q_{1}$ and $q_{2}$ are quadratic polynomials. In order to lie in $\mathbf{R} \cap \mathbf{E}, F$ must be of the form $f_{*} \mathcal{O}_{\tilde{C}}$, with $p$ a singular point of $C$. By the classification of sheaves in $\mathbf{E}^{\infty}$ found at Proposition 3.4 we see that case (1) occurs precisely when $\operatorname{Ext}^{1}\left(\mathbb{C}_{p}, \mathcal{O}_{C}\right) \simeq \mathbb{C}$. Indeed, we may take $\tilde{C}$ to be the partial normalization of $C$ at $p$. The same argument applies in case (3). In case (2) we may take two different partial normalizations of $C$ at $p$, each yielding a sheaf in $\mathbf{R} \cap \mathbf{E}$.

Note that, in case (2), by Proposition 4.6(1) there is a nonstable sheaf in the extension $\operatorname{Ext}^{1}\left(\mathbb{C}_{p}, \mathcal{O}_{C}\right)$. By the properness of the moduli space the flat limit of sheaves in this extension must lie in $W^{+}$and hence outside of $\mathbf{R}$. We will see below that in the case of Proposition 4.6(2) the flat limit of sheaves in the said extension is still in $\mathbf{R}$. This does not contradict Proposition 5.7 because in this case the flat limit is a planar sheaf and hence not in $W^{+}$.

COROLlary 5.9. In each of the following cases the sheaf $F$ gives a point in $\mathbf{R} \cap \mathbf{E}$ :

(1) $F=\mathcal{O}_{C}(p)$, where $C$ is a singular curve of type $(2,2)$ on a smooth quadric surface, and $p \in \operatorname{sing}(C)$;

(2) $F=\mathcal{O}_{C}(p)$, where $C$ is a quadruple line supported on $L$ (the intersection of a double plane containing $L$ with the union of two distinct planes each containing $L)$, and $p \in L$;

(3) $F=\mathcal{O}_{C}(p)$, where $C$ is the union of a singular planar curve $C_{0}$ with an incident line $L$, and $p \in \operatorname{sing}\left(C_{0}\right) \backslash L$.

Proof. The sheaves from (1) are limits of sheaves from Proposition 5.8(1)(a) because the set of singular curves of type $(2,2)$ on $\mathbb{P}^{1} \times \mathbb{P}^{1}$ is irreducible ([34, Thm. 3.1]). In particular, if $C$ is a double conic (meaning the intersection of a double plane with a smooth quadric $S$ ) and $p \in C$, then $\mathcal{O}_{C}(p) \in \mathbf{R} \cap \mathbf{E}$. The sheaves from (2) are limits of such sheaves (make $S$ converge to the union of two distinct planes). Finally, the sheaves from (3) are limits of sheaves from Proposition 5.8(1)(a).

We will next examine the case where the support of the sheaf is the union of a double line and a conic. The picture becomes more complicated. Case (1) of Proposition 5.10 was already dealt with at Corollary 5.9(1), but we treat it also using modifications of sheaves in order to better illustrate the argument.

Proposition 5.10. In each of the following cases, all stable sheaves $F \in$ $\mathbb{P}\left(\operatorname{Ext}^{1}\left(\mathbb{C}_{p}, \mathcal{O}_{C}\right)\right)$ belong to $\mathbf{R} \cap \mathbf{E}$ :

(1) $C=L^{2} Q$ is a union of a double line on $L$ and a conic $Q$ with $L$ and $Q$ meeting at a point, and $p \in L$;

(2) $C=L^{2} Q$ is a union of a genus -2 double line $L^{2}$ and a conic $Q$, and $p \in$ $Q \cap L$. 
Proof. (1) We can see that the unique extension sheaf $F \in \operatorname{Ext}^{1}\left(\mathbb{C}_{p}, \mathcal{O}_{C}\right)=\mathbb{C}$ fits into the exact sequence

$$
0 \rightarrow \mathcal{O}_{L}(-1) \rightarrow F \rightarrow \mathcal{O}_{L \cup Q} \rightarrow 0
$$

by using the isomorphism $\operatorname{Ext}^{1}\left(\mathbb{C}_{p}, \mathcal{O}_{L}(-2)\right) \simeq \operatorname{Ext}^{1}\left(\mathbb{C}_{p}, \mathcal{O}_{C}\right)$, which is given by the exact sequence $0 \rightarrow \mathcal{O}_{L}(-2) \rightarrow \mathcal{O}_{C} \rightarrow \mathcal{O}_{L \cup Q} \rightarrow 0$. Also by direct computation, stable sheaves in (10) are parameterized by $\operatorname{Ext}^{1}\left(\mathcal{O}_{L \cup Q}, \mathcal{O}_{L}(-1)\right)=\mathbb{C}^{3}$. We prove that the sheaves in (10) arise as elementary modifications of sheaves by using finite maps. Let $f: C \rightarrow \mathbb{P}^{3}$ be a map whose domain $C$ is the pair of two lines $L_{1} \cup L_{2}$ such that $f_{\mid L_{1}}$ is a degree two map onto $L$ and $f_{\mid L_{2}}$ is a bijection onto $Q$. Then obviously $f$ can be regarded as an element in the moduli space $F_{0}\left(\mathbb{P}^{3}, 4\right)$. Let $\Delta \subset F_{0}\left(\mathbb{P}^{3}, 4\right)$ be the locus of the finite maps whose image is the union of lines and smooth conics meeting at a point. Then, the direct image sheaf $f_{*} \mathcal{O}_{C}$ fits into the exact sequence

$$
0 \rightarrow \mathcal{O}_{L \cup Q} \rightarrow f_{*} \mathcal{O}_{C} \rightarrow \mathcal{O}_{L}(-1) \rightarrow 0 .
$$

We show by modifications of sheaves that all sheaves in (10) lie in R. For example, see [9]. To apply the modification technique, we need to choose a smooth chart of $F_{0}\left(\mathbb{P}^{3}, 4\right)$ at $f$. Around $f$, by $[29$, Thm. 0.1$]$, the maps space $F_{0}\left(\mathbb{P}^{3}, 4\right)$ can be obtained as the $S L(2)$-quotient of the moduli space $F_{0}\left(\mathbb{P}^{1} \times \mathbb{P}^{3},(1,4)\right)$ of finite maps in $\mathbb{P}^{1} \times \mathbb{P}^{3}$ of bidegree $(1,4)$, where $\operatorname{Aut}\left(\mathbb{P}^{1}\right)=S L(2)$ canonically acts on $F_{0}\left(\mathbb{P}^{1} \times \mathbb{P}^{3},(1,4)\right)$. Among the fibers $f$ along the GIT-quotient map, let us choose the graph map $f^{\prime}$ whose restriction on $L_{1}$ is of bidegree $(1,2)$ and which doubly covers $\mathbb{P}^{1} \times L \subset \mathbb{P}^{1} \times \mathbb{P}^{3}$; then $f^{\prime}$ has only the trivial automorphism. Hence, around $f$, the space $F_{0}\left(\mathbb{P}^{1} \times \mathbb{P}^{3},(1,4)\right)$ is a smooth chart that is compatible with $S L(2)$-action. This implies that the argument in [9, Lemma 4.6] about the construction of the Kodaira-Spencer map of the maps space can be naturally applied in our setting. Now, let us compute the normal space by the same technique as in [9]. We write $f$ as a composition $C \stackrel{h}{\rightarrow} C^{\prime} \stackrel{g}{\rightarrow} \mathbb{P}^{3}$, where $C^{\prime}$ is a pair of lines. Here $h$ is a two-to-one covering from $L_{1}$ and a bijection from $L_{2}$. Also, $g$ is an isomorphism of degree 3. By the octahedron axiom for the derived category we have the exact sequence

$$
\begin{aligned}
0 & \rightarrow \operatorname{Def}(h) \rightarrow \operatorname{Def}(f) \rightarrow \operatorname{Ext}^{1}\left(h^{*}\left[g^{*} \Omega_{\mathbb{P}^{3}} \rightarrow \Omega_{C^{\prime}}\right], \mathcal{O}_{C}\right) \\
& \rightarrow \operatorname{Ob}(h) \simeq \operatorname{Ext}^{1}\left(\Omega_{C}, \mathcal{O}_{C}\right) \rightarrow 0 .
\end{aligned}
$$

Since the higher direct images $R^{\bullet} h_{*}(-)$ vanish, we have an isomorphism

$$
\operatorname{Ext}^{1}\left(h^{*}\left[g^{*} \Omega_{\mathbb{P}^{3}} \rightarrow \Omega_{C^{\prime}}\right], \mathcal{O}_{C}\right) \simeq \operatorname{Ext}^{1}\left(\left[g^{*} \Omega_{\mathbb{P}^{3}} \rightarrow \Omega_{C^{\prime}}\right], h_{*} \mathcal{O}_{C}\right) .
$$

But the later space is decomposed into

$$
\begin{aligned}
0 & \rightarrow \operatorname{Ext}^{1}\left(\left[g^{*} \Omega_{\mathbb{P}^{3}} \rightarrow \Omega_{C^{\prime}}\right], \mathcal{O}_{C^{\prime}}\right) \rightarrow \operatorname{Ext}^{1}\left(\left[g^{*} \Omega_{\mathbb{P}^{3}} \rightarrow \Omega_{C^{\prime}}\right], h_{*} \mathcal{O}_{C}\right) \\
& \rightarrow \operatorname{Ext}^{1}\left(\left[g^{*} \Omega_{\mathbb{P}^{3}} \rightarrow \Omega_{C^{\prime}}\right], O_{L}(-1)\right)
\end{aligned}
$$

by the exact sequence

$$
0 \rightarrow \mathcal{O}_{C^{\prime}} \rightarrow h_{*} \mathcal{O}_{C} \rightarrow \mathcal{O}_{L}(-1) \rightarrow 0 .
$$


The first term in (12) is the deformation space of $g$ in $\mathbb{P}^{3}$. By using the local isomorphism $F_{0}\left(\mathbb{P}^{3}, 3\right) \simeq \operatorname{Hilb}_{\mathbb{P}^{3}}(3 m+1)$ around $g$ associating $g$ to $g_{*} \mathcal{O}_{C^{\prime}}=$ $\mathcal{O}_{L \cup Q}([9$, Thm. 1.4 and Prop. 3.3 (1)]) we can easily see that there exists a natural surjective homomorphism

$$
\begin{aligned}
\psi: \operatorname{Ext}^{1}\left(\left[g^{*} \Omega_{\mathbb{P}^{3}} \rightarrow \Omega_{C^{\prime}}\right], \mathcal{O}_{C^{\prime}}\right) & \simeq \operatorname{Hom}\left(I_{L \cup Q}, \mathcal{O}_{L \cup Q}\right) \\
& \rightarrow \operatorname{Ext}^{1}\left(\Omega_{L \cup Q}, \mathcal{O}_{L \cup Q}\right) \simeq \mathbb{C},
\end{aligned}
$$

where the map comes from the short exact sequence

$$
0 \rightarrow I_{L \cup Q} /\left.I_{L \cup Q}^{2} \rightarrow \Omega_{\mathbb{P}^{3}}\right|_{L \cup Q} \rightarrow \Omega_{L \cup Q} \rightarrow 0 .
$$

The kernel of $\psi$ is the deformation space of the map $g$ while fixing the node $p$ of the curve $L \cup Q$. In conclusion, we have a commutative diagram

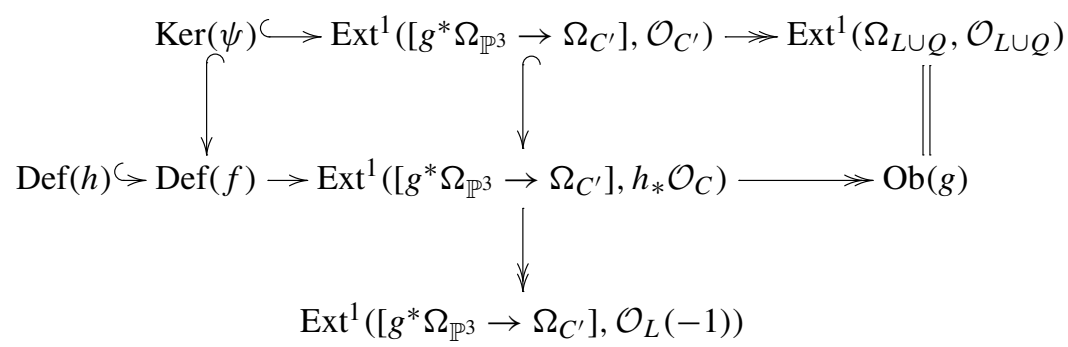

Combining with the deformation space $\operatorname{Def}(h) \simeq \mathbb{C}^{2}$ ([9, Lemma 4.10]), we see that the normal space of $\Delta$ in $F_{0}\left(\mathbb{P}^{3}, 4\right)$ at $f$ is isomorphic to

$$
N_{\Delta / F_{0}\left(\mathbb{P}^{3}, 4\right), f} \simeq \operatorname{Ext}^{1}\left(\left[g^{*} \Omega_{\mathbb{P}^{3}} \rightarrow \Omega_{C^{\prime}}\right], \mathcal{O}_{L}(-1)\right) .
$$

Since the curve $L \cup Q$ is a locally complete intersection, the two-term complex $\left[g^{*} \Omega_{\mathbb{P}^{3}} \rightarrow \Omega_{C^{\prime}}\right]$ is quasi-isomorphic to $g^{*} N_{L \cup Q / \mathbb{P}^{3}}^{*}[1]$. Thus,

$$
N_{\Delta / F_{0}\left(\mathbb{P}^{3}, 4\right), f} \simeq \operatorname{Ext}^{1}\left(N_{L \cup Q / \mathbb{P}^{3}}^{*}[1], \mathcal{O}_{L}(-1)\right)=\operatorname{Hom}\left(N_{L \cup Q / \mathbb{P}^{3}}^{*}, \mathcal{O}_{L}(-1)\right) .
$$

Since the choice of the normal vectors in the tangent space of maps space at $f$ makes the switch of the sub/quotient sheaf of the original sheaf in (11) (see [10]), the Kodaira-Spencer map $T_{f} F_{0}\left(\mathbb{P}^{3}, 4\right) \rightarrow \operatorname{Ext}^{1}\left(f_{*} \mathcal{O}_{C}, f_{*} \mathcal{O}_{C}\right)$ descents to

$$
N_{\Delta / F_{0}\left(\mathbb{P}^{3}, 4\right), f}=\operatorname{Hom}\left(N_{L \cup Q / \mathbb{P}^{3}}^{*}, \mathcal{O}_{L_{0}}(-1)\right) \simeq \operatorname{Ext}^{1}\left(\mathcal{O}_{L \cup Q}, \mathcal{O}_{L_{0}}(-1)\right),
$$

which is the compatible with the coboundary map given by the structure sequence

$$
0 \rightarrow I_{L \cup Q} \rightarrow \mathcal{O} \rightarrow \mathcal{O}_{L \cup Q} \rightarrow 0 .
$$

Therefore, the normal directions to $\Delta$ correspond exactly to the sheaves in the extension (10). This shows that such sheaves are obtained by elementary modifications and hence lie in $\mathbf{R}$.

(2) Similar arguments as before can be applied. Again, we want to show that all sheaves in the extension

$$
0 \rightarrow \mathcal{O}_{L}(-1) \rightarrow F \rightarrow \mathcal{O}_{L \cup Q}(p) \rightarrow 0
$$


lie in $\mathbf{R}$. Recall that in our convention $\mathcal{O}_{L \cup Q}(p)$ is the unique nonsplit extension of $\mathbb{C}_{p}$ by $\mathcal{O}_{L \cup Q}$. First, let us fix the point $p \in \mathbb{P}^{3}$. Let $\Delta_{1,3}$ be the locus of stable maps $f: L_{1} \cup L_{3} \rightarrow \mathbb{P}^{3}$ such that degrees of $f$ on $L_{1}$ and $L_{3}$ are 1 and 3, respectively, and $f$ maps $L_{1} \cap L_{3}$ to $p$. Let $D$ be the locus in the boundary of $\Delta_{1,3}$ consisting of the finite maps $f: L_{1} \cup\left(L_{1}^{\prime} \cup L_{2}\right) \rightarrow \mathbb{P}^{3}$ such that the image $\left.f\right|_{L_{1}^{\prime} \cup L_{2}}$ is the union of a line $L$ and a coplanar conic and the restriction $\left.f\right|_{L_{1}}$ is a bijection with the line $L$. Then the two spaces are smooth around $f$.

Let us describe the restricted Kodaira-Spencer map

$$
\xi: N_{D / \Delta_{1,3}, f} \rightarrow \operatorname{Ext}_{\mathbb{P}^{3}}^{1}\left(\mathcal{O}_{L \cup Q}(p), \mathcal{O}_{L}(-1)\right) .
$$

The normal space of $D$ in $\Delta_{1,3}$ consists of the two-dimensional contribution from smoothing two nodes of $L \cup Q$ and the two-dimensional contribution from moving $L$ away from the plane containing $Q$. By definition, $p$ is one node. We let $q$ be the other node. From the exact sequence

$$
0 \rightarrow \mathcal{O}_{Q}(-q) \rightarrow \mathcal{O}_{L \cup Q}(p) \rightarrow \mathcal{O}_{L} \rightarrow 0
$$

we have

$$
\begin{aligned}
0 & \rightarrow \operatorname{Ext}_{\mathbb{P}^{3}}^{1}\left(\mathcal{O}_{L}, \mathcal{O}_{L}(-1)\right) \rightarrow \operatorname{Ext}_{\mathbb{P}^{3}}^{1}\left(\mathcal{O}_{L \cup Q}(p), \mathcal{O}_{L}(-1)\right) \\
& \rightarrow \operatorname{Ext}_{\mathbb{P}^{3}}^{1}\left(\mathcal{O}_{Q}(-q), \mathcal{O}_{L}(-1)\right) \rightarrow 0 .
\end{aligned}
$$

The first term

$$
\operatorname{Ext}_{\mathbb{P}^{3}}^{1}\left(\mathcal{O}_{L}, \mathcal{O}_{L}(-1)\right) \simeq \mathrm{H}^{0}\left(N_{L / \mathbb{P}^{3}}(-1)\right) \simeq \mathbb{C}^{2}
$$

is the deformation of $L$ while fixing the point $p$. The third term

$$
\operatorname{Ext}_{\mathbb{P}^{3}}^{1}\left(\mathcal{O}_{Q}(-q), \mathcal{O}_{L}(-1)\right) \simeq \operatorname{Ext}_{L}^{1}\left(\mathbb{C}_{p} \oplus \mathbb{C}_{q}, \mathcal{O}_{L}(-1)\right) \simeq \mathbb{C} \oplus \mathbb{C}
$$

is the deformation space of smoothing the two nodes of the plane cubic curve $L \cup Q$ in the moduli space $\operatorname{Hilb}_{\mathbb{P}^{3}}(3 m+1)$ while fixing the point $p$. After a diagram chase, we check that $\xi$ is an isomorphism, and thus the modified sheaf lies in $\mathbf{R}$. Thus, we see that all sheaves in (13) belong to $\mathbf{R}$.

Finally, we show that $\mathbf{R} \cap \mathbf{E}$ is irreducible. In fact, $\mathbf{R} \cap \mathbf{E}$ is the closure of the locus of sheaves from Proposition 5.8(1)(a). Since it is straightforward that all other loci of $\mathbf{R} \cap \mathbf{E}$ are in the closure, we will show the sheaves from Propostion 5.10(2) do not form a new irreducible component. We denote this locus by $\mathbf{E}_{2 b}$ following the notation in the previous section. We remark that $\mathbf{E}_{2 b}$ is irreducible.

Lemma 5.11. If $F \in \mathbf{E}_{2 b}$ is generic, then $\operatorname{ext}^{1}(F, F) \leq 19$.

Proof. Since $\mathbf{E}_{2 b}$ is irreducible, it is enough to prove the estimate for a single sheaf. Let $F$ be given by the resolution

$$
0 \rightarrow 3 \mathcal{O}(-3) \stackrel{\psi}{\rightarrow} 5 \mathcal{O}(-2) \stackrel{\varphi}{\rightarrow} \mathcal{O}(-1) \oplus \mathcal{O} \rightarrow F \rightarrow 0,
$$




$$
\psi=\left[\begin{array}{ccc}
-y & -z & 0 \\
x & 0 & 0 \\
0 & x & 0 \\
0 & -y & x \\
0 & 0 & -y
\end{array}\right], \quad \varphi=\left[\begin{array}{ccccc}
x & y & z & 0 & 0 \\
0 & 0 & y^{2} & x y & x^{2}
\end{array}\right] .
$$

This resolution is one of three possible resolution types we will encounter in Section 6. The support of $F$ is the subscheme defined by the ideal $\left\langle x^{2}, x y, y^{3}\right\rangle$, and the point $p$ is defined by $\langle x, y, z\rangle$. Thus, $F$ lies in $\mathbf{E}_{2 b}$. It is easy to check, with the help of the computer program Macaulay2 ([13]), that $\operatorname{Ext}^{1}(F, F) \cong \mathbb{C}^{19}$.

Theorem 5.12. The variety $\mathbf{R} \cap \mathbf{E}$ is irreducible.

Proof. Suppose that the variety $\mathbf{E}_{2 b} \subset \mathbf{R} \cap \mathbf{E}$ forms a new irreducible component. Choose a regular point $y$ of $\mathbf{E}_{2 b}$. Then

$$
\begin{aligned}
\operatorname{dim} T_{y} \mathbf{M}_{\mathbb{P}^{3}}(4 m+1) & \geq \operatorname{dim} T_{y} \mathbf{R}+\operatorname{dim} T_{y} \mathbf{E}-\operatorname{dim} T_{y} \mathbf{E}_{2 b} \\
& \geq 16+17-13=20 .
\end{aligned}
$$

Since we may choose $y$ to be generic, this contradicts the estimate at Lemma 5.11.

\section{The Resolutions of the Sheaves in $\mathrm{M}(4 m+1)$}

We fix a four-dimensional vector space $V$ over $\mathbb{C}$ and a basis $\{X, Y, Z, W\}$ of $V^{*}$. We identify $\mathbb{P}^{3}$ with $\mathbb{P}(V)$. Let $F$ be a one-dimensional sheaf on $\mathbb{P}^{3}$. The relevant part of the $E^{1}$-level of the Beilinson spectral sequence ([35, Thm. 3.1.4]) converging to $F$ is displayed in the following tableau:

$$
\begin{aligned}
& \mathrm{H}^{1}(F(-1)) \otimes \mathcal{O}(-3) \stackrel{\varphi_{1}}{\longrightarrow} \mathrm{H}^{1}\left(F \otimes \Omega^{2}(2)\right) \otimes \mathcal{O}(-2) \\
& \stackrel{\varphi_{2}}{\longrightarrow} \mathrm{H}^{1}\left(F \otimes \Omega^{1}(1)\right) \otimes \mathcal{O}(-1) \stackrel{\varphi_{3}}{\longrightarrow} \mathrm{H}^{1}(F) \otimes \mathcal{O}, \\
& \mathrm{H}^{0}(F(-1)) \otimes \mathcal{O}(-3) \stackrel{\varphi_{4}}{\longrightarrow} \mathrm{H}^{0}\left(F \otimes \Omega^{2}(2)\right) \otimes \mathcal{O}(-2) \\
& \stackrel{\varphi_{5}}{\longrightarrow} \mathrm{H}^{0}\left(F \otimes \Omega^{1}(1)\right) \otimes \mathcal{O}(-1) \stackrel{\varphi_{6}}{\longrightarrow} \mathrm{H}^{0}(F) \otimes \mathcal{O} .
\end{aligned}
$$

The $E^{2}$-level has the tableau

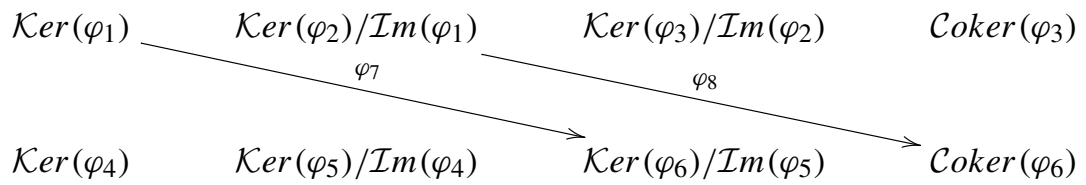

The spectral sequence degenerates at $E^{3}$, where all maps are zero:

$$
\operatorname{Ker}\left(\varphi_{7}\right) \quad \mathcal{K} \operatorname{er}\left(\varphi_{8}\right) \quad \mathcal{K} \operatorname{er}\left(\varphi_{3}\right) / \mathcal{I} m\left(\varphi_{2}\right) \quad \operatorname{Coker}\left(\varphi_{3}\right)
$$

$\operatorname{Ker}\left(\varphi_{4}\right) \quad \mathcal{K} \operatorname{er}\left(\varphi_{5}\right) / \mathcal{I} m\left(\varphi_{4}\right) \quad \operatorname{Coker}\left(\varphi_{7}\right) \quad \operatorname{Coker}\left(\varphi_{8}\right)$ 
Thus $\varphi_{7}$ is an isomorphism, $\varphi_{3}$ is surjective, $\varphi_{4}$ is injective, $\operatorname{Ker}\left(\varphi_{5}\right)=\operatorname{Im}\left(\varphi_{4}\right)$, and we have the exact sequence

$$
0 \rightarrow \operatorname{Ker}\left(\varphi_{2}\right) / \operatorname{I} m\left(\varphi_{1}\right) \stackrel{\varphi_{8}}{\longrightarrow} \operatorname{Coker}\left(\varphi_{6}\right) \rightarrow F \rightarrow \operatorname{Ker}\left(\varphi_{3}\right) / \mathcal{I} m\left(\varphi_{2}\right) \rightarrow 0 .
$$

Denote $p=\mathrm{h}^{0}\left(F \otimes \Omega^{1}(1)\right), q=\mathrm{h}^{0}\left(F \otimes \Omega^{2}(2)\right)$. Assume that $F$ is semistable and has Hilbert polynomial $P_{F}(m)=4 m+1$. According to [6], we have the relations

$$
\mathrm{h}^{0}(F(-1))=0, \quad \mathrm{~h}^{1}(F)=0 \text { or } 1 .
$$

The Beilinson monad with middle cohomology $F$ yields an exact sequence

$$
\begin{aligned}
0 & \rightarrow 3 \mathcal{O}(-3) \oplus q \mathcal{O}(-2) \stackrel{\psi}{\rightarrow}(q+5) \mathcal{O}(-2) \oplus p \mathcal{O}(-1) \\
& \stackrel{\varphi}{\rightarrow} \operatorname{Ker}\left(\varphi_{3}\right) \oplus \mathrm{H}^{0}(F) \otimes \mathcal{O} \rightarrow F \rightarrow 0,
\end{aligned}
$$

in which $\psi_{12}=0$ and $\varphi_{12}=0$. We recall two well-known facts. The sheaves giving points in $\mathbf{M}_{\mathbb{P}^{3}}(m+1)$ are precisely the structure sheaves of lines. The sheaves giving points in $\mathbf{M}_{\mathbb{P}^{3}}(2 m+1)$ are precisely the structure sheaves of conic curves.

THEOREM 6.1. Let $F$ give a point in $\mathrm{M}_{\mathbb{P}^{3}}(4 m+1)$. Then precisely one of the following is true:

(i) $\mathrm{h}^{0}\left(F \otimes \Omega^{2}(2)\right)=0, \mathrm{~h}^{0}\left(F \otimes \Omega^{1}(1)\right)=0, \mathrm{~h}^{0}(F)=1$;

(ii) $\mathrm{h}^{0}\left(F \otimes \Omega^{2}(2)\right)=0, \mathrm{~h}^{0}\left(F \otimes \Omega^{1}(1)\right)=1, \mathrm{~h}^{0}(F)=1$;

(iii) $\mathrm{h}^{0}\left(F \otimes \Omega^{2}(2)\right)=1, \mathrm{~h}^{0}\left(F \otimes \Omega^{1}(1)\right)=3, \mathrm{~h}^{0}(F)=2$.

The sheaves satisfying conditions (i) are precisely the sheaves having a resolution of the form

$$
\begin{aligned}
0 & \rightarrow 3 \mathcal{O}(-3) \stackrel{\psi}{\rightarrow} 5 \mathcal{O}(-2) \stackrel{\varphi}{\rightarrow} \mathcal{O}(-1) \oplus \mathcal{O} \rightarrow F \rightarrow 0, \\
\varphi & =\left[\begin{array}{lllll}
l_{1} & l_{2} & l_{3} & l_{4} & l_{5} \\
q_{1} & q_{2} & q_{3} & q_{4} & q_{5}
\end{array}\right],
\end{aligned}
$$

where $\operatorname{dim}\left(\operatorname{span}\left\{l_{1}, l_{2}, l_{3}, l_{4}, l_{5}\right\}\right) \geq 3$.

The sheaves satisfying conditions (ii) are precisely the sheaves having a resolution of the form

$$
0 \rightarrow 3 \mathcal{O}(-3) \stackrel{\psi}{\rightarrow} 5 \mathcal{O}(-2) \oplus \mathcal{O}(-1) \stackrel{\varphi}{\rightarrow} 2 \mathcal{O}(-1) \oplus \mathcal{O} \rightarrow F \rightarrow 0,
$$

where $\varphi_{12}=0$, and $\varphi_{11}: 5 \mathcal{O}(-2) \rightarrow 2 \mathcal{O}(-1)$ is not equivalent to a morphism of the form

$$
\left[\begin{array}{lcccc}
\star & \star & 0 & 0 & 0 \\
\star & \star & \star & \star & \star
\end{array}\right] \text { or }\left[\begin{array}{ccccc}
\star & \star & \star & \star & 0 \\
\star & \star & \star & \star & 0
\end{array}\right] \text {. }
$$

The sheaves satisfying conditions (iii) are precisely the sheaves having a resolution of the form

$$
0 \rightarrow \mathcal{O}(-4) \oplus \mathcal{O}(-2) \stackrel{\psi}{\rightarrow} \mathcal{O}(-3) \oplus 3 \mathcal{O}(-1) \stackrel{\varphi}{\rightarrow} 2 \mathcal{O} \rightarrow F \rightarrow 0,
$$




$$
\psi=\left[\begin{array}{cc}
l & 0 \\
0 & l \\
-f_{1} & -l_{1} \\
-f_{2} & -l_{2}
\end{array}\right], \quad \varphi=\left[\begin{array}{cccc}
f_{1} & l_{1} & l & 0 \\
f_{2} & l_{2} & 0 & l
\end{array}\right],
$$

where $l, l_{1}, l_{2}$ are linearly independent one-forms. If $H \subset \mathbb{P}^{3}$ is the plane given by the equation $l=0$, then $F$ has resolution

$$
\begin{aligned}
0 & \rightarrow \mathcal{O}_{H}(-3) \oplus \mathcal{O}_{H}(-1) \stackrel{\bar{\varphi}}{\rightarrow} 2 \mathcal{O}_{H} \rightarrow F \rightarrow 0, \\
\bar{\varphi} & =\left[\begin{array}{ll}
\bar{f}_{1} & \bar{l}_{1} \\
\bar{f}_{2} & \bar{l}_{2}
\end{array}\right],
\end{aligned}
$$

where $\bar{f}_{1}, \bar{f}_{2}, \bar{l}_{1}, \bar{l}_{2}$ denote classes modulo $l$.

Proof. (i) Assume first that $\mathrm{h}^{0}(F)=1$. The exact sequence (16) becomes

$$
\begin{aligned}
0 \rightarrow 3 \mathcal{O}(-3) \oplus q \mathcal{O}(-2) \stackrel{\psi}{\rightarrow}(q+5) \mathcal{O}(-2) \oplus p \mathcal{O}(-1) \\
\quad \stackrel{\varphi}{\rightarrow}(p+1) \mathcal{O}(-1) \oplus \mathcal{O} \rightarrow F \rightarrow 0 .
\end{aligned}
$$

We claim that $p=0$ or 1 . Indeed, if $p=2$, then we would get a commutative diagram

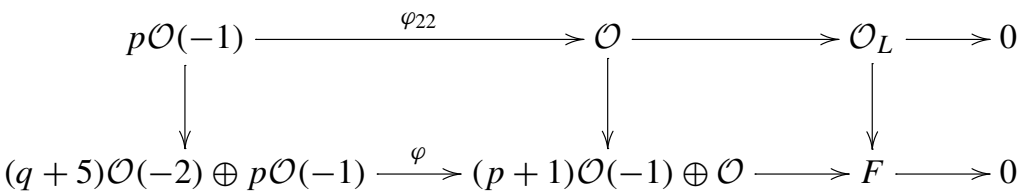

Both $\mathcal{O}_{L}$ and $F$ are stable, and $\mathrm{p}\left(\mathcal{O}_{L}\right)=1>\mathrm{p}(F)$; hence, $\operatorname{Hom}\left(\mathcal{O}_{L}, F\right)=0$. Thus, $\mathcal{O} \rightarrow F$ is the zero morphism. On the other hand, $\mathrm{H}^{0}(\mathcal{O}) \rightarrow \mathrm{H}^{0}(F)$ is injective because $\mathrm{H}^{0}(\operatorname{Coker}(\psi))=0$. We have obtained a contradiction. If $p=3$ or $p \geq 4$, then $\operatorname{Coker}\left(\varphi_{22}\right)$ would be the structure sheaf of a point, respectively, it would be zero. Both cases would yield contradictions as before.

Assume that $p=0$. Then $q=0$ because $\varphi_{5}$ is injective. We obtain the resolution

$$
\begin{aligned}
0 & \rightarrow 3 \mathcal{O}(-3) \rightarrow 5 \mathcal{O}(-2) \stackrel{\varphi}{\rightarrow} \mathcal{O}(-1) \oplus \mathcal{O} \rightarrow F \rightarrow 0, \\
\varphi & =\left[\begin{array}{lllll}
l_{1} & l_{2} & l_{3} & l_{4} & l_{5} \\
q_{1} & q_{2} & q_{3} & q_{4} & q_{5}
\end{array}\right] .
\end{aligned}
$$

If $\operatorname{dim}\left(\operatorname{span}\left\{l_{1}, l_{2}, l_{3}, l_{4}, l_{5}\right\}\right)=1$, then we may assume that $l_{1} \neq 0$ and that $l_{2}, l_{3}$, $l_{4}, l_{5}$ are zero. We would get a commutative diagram

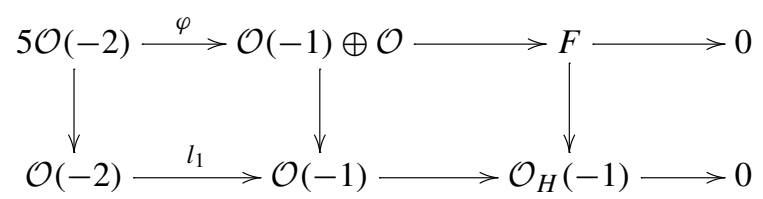

showing that $F$ maps surjectively to $\mathcal{O}_{H}(-1)$. This is absurd because $\operatorname{dim}(\operatorname{supp}(F))=1$. Likewise, if $\operatorname{dim}\left(\operatorname{span}\left\{l_{1}, l_{2}, l_{3}, l_{4}, l_{5}\right\}\right)=2$, then $F$ would 
have a quotient sheaf of the form $\mathcal{O}_{L}(-1)$, in violation of semistability. We conclude that $F$ has resolution (17).

Conversely, we assume that $F$ has resolution (17), and we must show that $F$ is semistable. At every point $P \in \mathbb{P}^{3}$, we have $\operatorname{hd}_{P}(F) \leq 2$, and hence $\operatorname{depth}_{P}(F) \geq 1$. From Grothendieck's criterion we deduce that $\mathcal{H}_{\{P\}}^{0}(F)=0$, that is, $F$ has no sections supported on $\{P\}$. Thus, $F$ has no zero-dimensional torsion. Assume that $F$ had a destabilizing subsheaf $E$. We may assume that $E$ is semistable. Since $\mathrm{h}^{0}(E) \leq \mathrm{h}^{0}(F)=1$, the Hilbert polynomial of $E$ may be one of the following: $m+1,2 m+1,3 m+1$. In the first case, $E \simeq \mathcal{O}_{L}$, and its standard resolution fits into a commutative diagram

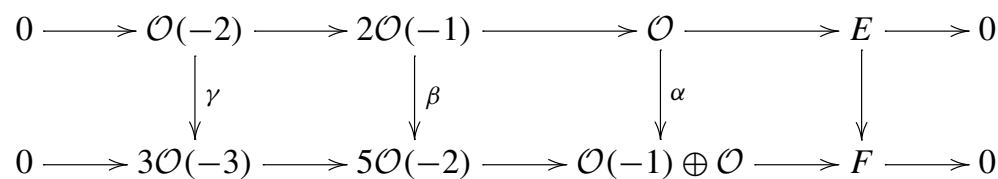

Since $\alpha \neq 0$, we have $\mathcal{K} \operatorname{er}(\alpha)=0$, and hence $\mathcal{K} \operatorname{er}(\gamma) \simeq \mathcal{K} \operatorname{er}(\beta)=2 \mathcal{O}(-1)$. This is absurd. In the second case, $E$ is the structure sheaf of a conic curve, and its standard resolution fits into a commutative diagram

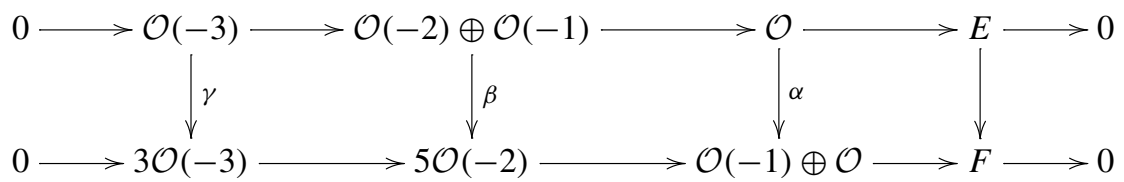

Since $\alpha \neq 0$, we have $\mathcal{K} \operatorname{er}(\gamma) \simeq \mathcal{K} \operatorname{er}(\beta)$. It follows that $\mathcal{O}(-1)$ is a subsheaf of $\operatorname{Ker}(\gamma)$. This is absurd. Finally, assume that $P_{E}(m)=3 m+1$. The quotient $G=F / E$ has no zero-dimensional torsion, and $P_{G}(m)=m$. It follows that $G \simeq$ $\mathcal{O}_{L}(-1)$. We have a commutative diagram

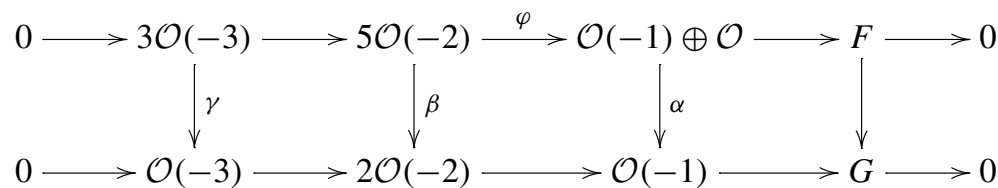

From the commutativity of the middle square we see that

$$
\varphi \sim\left[\begin{array}{ccccc}
\star & \star & 0 & 0 & 0 \\
\star & \star & \star & \star & \star
\end{array}\right] .
$$

This contradicts our hypothesis. We conclude that there are no destabilising subsheaves $E \subset F$.

(ii) We next examine the case where $\mathrm{h}^{0}\left(F \otimes \Omega^{1}(1)\right)=1$ and $\mathrm{h}^{0}(F)=1$. Since $\varphi_{5}$ is injective, we see that $q=0$ or 1 . If $q=1$, then $\varphi_{6}$ would be generically zero, hence $\varphi_{6}=0$, and hence $\operatorname{Ker}\left(\varphi_{6}\right) / \mathcal{I} m\left(\varphi_{5}\right) \simeq \mathcal{O}_{H}(-1)$. Recall that $\varphi_{7}: \mathcal{K} \operatorname{er}\left(\varphi_{1}\right) \rightarrow \operatorname{K} \operatorname{er}\left(\varphi_{6}\right) / \mathcal{I} m\left(\varphi_{5}\right)$ is an isomorphism. It would follow that $\mathcal{O}_{H}(-1)$ is a subsheaf of $3 \mathcal{O}(-3)$. This is absurd. Thus, $q=0$, and we have a 
resolution

$$
0 \rightarrow 3 \mathcal{O}(-3) \rightarrow 5 \mathcal{O}(-2) \oplus \mathcal{O}(-1) \stackrel{\varphi}{\rightarrow} 2 \mathcal{O}(-1) \oplus \mathcal{O} \rightarrow F \rightarrow 0 .
$$

If $\varphi_{11}$ were equivalent to a morphism of the form

$$
\left[\begin{array}{lllll}
\star & \star & 0 & 0 & 0 \\
\star & \star & \star & \star & \star
\end{array}\right],
$$

then $F$ would have a quotient sheaf of the form $\mathcal{O}_{H}(-1)$ or of the form $\mathcal{O}_{L}(-1)$. This, as we saw before, yields a contradiction. If $\varphi$ were equivalent to a morphism of the form

$$
\left[\begin{array}{llllll}
\star & \star & \star & \star & 0 & 0 \\
\star & \star & \star & \star & 0 & 0 \\
\star & \star & \star & \star & q & l
\end{array}\right],
$$

then we would have a commutative diagram

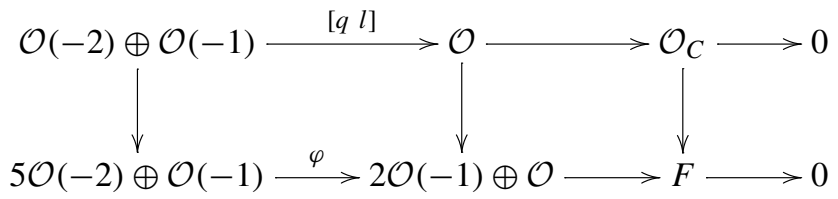

in which $C$ is the conic curve given by the equations $q=0, l=0$. Both $\mathcal{O}_{C}$ and $F$ are stable with $\mathrm{p}\left(\mathcal{O}_{C}\right)=1 / 2>\mathrm{p}(F)$, and hence $\operatorname{Hom}\left(\mathcal{O}_{C}, F\right)=0$. Thus, the map $\mathcal{O} \rightarrow F$ is zero. This, as we saw before, yields a contradiction. We conclude that $F$ has resolution (18).

Conversely, if $F$ has resolution (18), then, by arguments analogous to the arguments in the case of resolution (17), we can show that $F$ is semistable.

(iii) Finally, we consider the case where $\mathrm{h}^{0}(F)=2$. Then $p \geq 3$, and resolution (16) takes the form

$$
\begin{aligned}
0 & \rightarrow 3 \mathcal{O}(-3) \oplus q \mathcal{O}(-2) \stackrel{\psi}{\rightarrow}(q+5) \mathcal{O}(-2) \oplus p \mathcal{O}(-1) \\
& \stackrel{\varphi}{\rightarrow} \Omega^{1} \oplus(p-3) \mathcal{O}(-1) \oplus 2 \mathcal{O} \rightarrow F \rightarrow 0 .
\end{aligned}
$$

The morphism $\varphi_{32}: p \mathcal{O}(-1) \rightarrow 2 \mathcal{O}$ cannot be equivalent to a morphism represented by a matrix of the form

$$
\left[\begin{array}{ccccc}
\star & \cdots & \star & 0 & 0 \\
\star & \cdots & \star & l_{1} & l_{2}
\end{array}\right] ;
$$

otherwise, we would have a commutative diagram

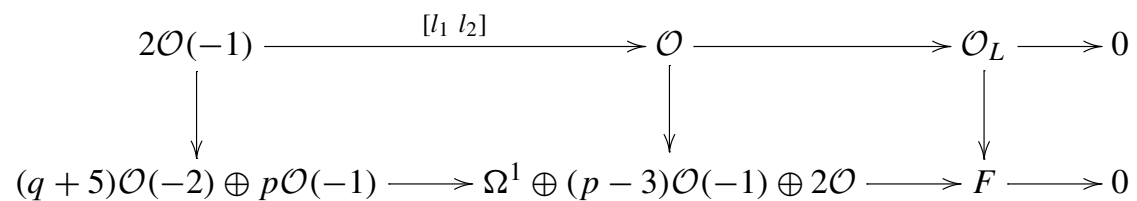

But $\operatorname{Hom}\left(\mathcal{O}_{L}, F\right)=0$, and hence the morphism $2 \mathcal{O} \rightarrow F$ is not injective. On the other hand, $\mathrm{H}^{0}(2 \mathcal{O}) \rightarrow \mathrm{H}^{0}(F)$ is injective because $\mathrm{H}^{0}(\operatorname{Coker}(\psi))=0$. This 
yields a contradiction. Thus, $p \leq 5$. Denote $E=\operatorname{Coker}\left(\varphi_{32}\right)$. Assume first that $p=5$. We may write

$$
\varphi_{32}=\left[\begin{array}{ccccc}
X & Y & Z & W & 0 \\
0 & l_{1} & l_{2} & l_{3} & l_{4}
\end{array}\right] .
$$

If $X$ and $l_{4}$ are linearly independent, then $E$ is supported on the line $L$ given by the equations $X=0, l_{4}=0$. Thus,

$$
E / \mathcal{T}^{0}(E) \simeq \mathcal{O}_{L}\left(d_{1}\right) \oplus \cdots \oplus \mathcal{O}_{L}\left(d_{n}\right) .
$$

Since $F$ is stable, $\operatorname{Hom}\left(\mathcal{O}_{L}(d), F\right)=0$ if $d \geq 0$. Thus, $\mathrm{H}^{0}(E) \rightarrow \mathrm{H}^{0}(F)$ is the zero morphism, and hence $\mathrm{H}^{0}(2 \mathcal{O}) \rightarrow \mathrm{H}^{0}(F)$ is also the zero morphism. This is a contradiction. We have reduced to the case where

$$
\varphi_{32}=\left[\begin{array}{ccccc}
X & Y & Z & W & 0 \\
0 & l_{1} & l_{2} & l_{3} & X
\end{array}\right],
$$

where $l_{1}, l_{2}, l_{3}$ are linearly independent one-forms in the variables $Y, Z, W$. Note that

$$
\left[\begin{array}{lll}
Y & Z & W \\
l_{1} & l_{2} & l_{3}
\end{array}\right] \nsim\left[\begin{array}{lll}
0 & \star & \star \\
\star & \star & \star
\end{array}\right]
$$

hence, the maximal minors of this matrix

$$
q_{1}=\left|\begin{array}{ll}
Z & W \\
l_{2} & l_{3}
\end{array}\right|, \quad q_{2}=\left|\begin{array}{ll}
Y & W \\
l_{1} & l_{3}
\end{array}\right|, \quad q_{3}=\left|\begin{array}{ll}
Y & Z \\
l_{1} & l_{2}
\end{array}\right|
$$

are linearly independent and have no common factor. It follows easily that there is an exact sequence

$$
0 \rightarrow \mathcal{O}(-4) \stackrel{\beta}{\rightarrow} \mathcal{O}(-3) \oplus 3 \mathcal{O}(-2) \stackrel{\alpha}{\rightarrow} 5 \mathcal{O}(-1) \stackrel{\varphi_{32}}{\rightarrow} 2 \mathcal{O} \rightarrow E \rightarrow 0,
$$

where

$$
\alpha=\left[\begin{array}{cccc}
0 & -Y & -Z & -W \\
q_{1} & X & 0 & 0 \\
-q_{2} & 0 & X & 0 \\
q_{3} & 0 & 0 & X \\
0 & -l_{1} & -l_{2} & -l_{3}
\end{array}\right], \quad \beta=\left[\begin{array}{llll}
-X & q_{1} & -q_{2} & q_{3}
\end{array}\right] .
$$

From this we get $P_{E}=3$, hence $\operatorname{Hom}(E, F)=0$, and hence $2 \mathcal{O}_{L} \rightarrow F$ is the zero morphism. This, as we saw before, yields a contradiction.

Assume now that $p=4$. We examine first the case where

$$
\varphi_{32} \sim\left[\begin{array}{cccc}
X & Y & Z & 0 \\
0 & l_{1} & l_{2} & l_{3}
\end{array}\right] .
$$

If $X$ and $l_{3}$ are linearly independent, then $E / \mathcal{T}^{0}(E)$ is supported on a line, and we get a contradiction as before. Thus, we may write

$$
\varphi_{32}=\left[\begin{array}{cccc}
X & Y & Z & 0 \\
0 & l_{1} & l_{2} & X
\end{array}\right],
$$


where $l_{1}$ and $l_{2}$ are linear forms in the variables $Y, Z, W$. It is easy to see that there is an exact sequence

$$
0 \rightarrow 2 \mathcal{O}(-2) \stackrel{\alpha}{\rightarrow} 4 \mathcal{O}(-1) \stackrel{\varphi_{32}}{\rightarrow} 2 \mathcal{O} \rightarrow E \rightarrow 0,
$$

where

$$
\alpha=\left[\begin{array}{cc}
-Y & -Z \\
X & 0 \\
0 & X \\
-l_{1} & -l_{2}
\end{array}\right] .
$$

We have that $P_{E}(m)=2 m+2$ and $E$ has no zero-dimensional torsion. From the semistability of $F$ we see that the morphism $E \rightarrow F$ is zero or it factors through a subsheaf $F^{\prime} \subset F$ with $P_{F^{\prime}}(m)=m-k, k \geq 0$. Thus, $\mathrm{H}^{0}\left(F^{\prime}\right)=0$, so, at any rate, $\mathrm{H}^{0}(E) \rightarrow \mathrm{H}^{0}(F)$ is the zero map. It follows that the map $\mathrm{H}^{0}(2 \mathcal{O}) \rightarrow \mathrm{H}^{0}(F)$ is zero, which yields a contradiction.

Assume next that

$$
\varphi_{32}=\left[\begin{array}{llll}
l_{11} & l_{12} & l_{13} & l_{14} \\
l_{21} & l_{22} & l_{23} & l_{24}
\end{array}\right] \nsim\left[\begin{array}{llll}
\star & \star & \star & 0 \\
0 & \star & \star & \star
\end{array}\right] .
$$

Then we may assume that

$$
\varphi^{\prime}=\left[\begin{array}{lll}
l_{11} & l_{12} & l_{13} \\
l_{21} & l_{22} & l_{23}
\end{array}\right] \nsim\left[\begin{array}{lll}
0 & \star & \star \\
\star & \star & \star
\end{array}\right] .
$$

Note that the maximal minors of $\varphi^{\prime}$ are linearly independent and have no common factor. According to [12] and [23], the sheaf $E^{\prime}=\operatorname{Coker}\left(\varphi^{\prime}\right)$ gives a point in $\mathrm{M}_{\mathbb{P}^{3}}(3 m+2)$. Note that $E$ is a quotient sheaf of $E^{\prime}$. Since $\operatorname{Hom}\left(E^{\prime}, F\right)=0$, it follows that $\operatorname{Hom}(E, F)=0$, and hence $2 \mathcal{O} \rightarrow F$ is the zero morphism. We have reached again a contradiction.

Thus far, we have proved that $p=3$. Resolution (16) takes the form

$$
0 \rightarrow 3 \mathcal{O}(-3) \oplus q \mathcal{O}(-2) \stackrel{\psi}{\rightarrow}(q+5) \mathcal{O}(-2) \oplus 3 \mathcal{O}(-1) \stackrel{\varphi}{\rightarrow} \Omega^{1} \oplus 2 \mathcal{O} \rightarrow F \rightarrow 0 .
$$

The morphism $\varphi_{22}: 3 \mathcal{O}(-1) \rightarrow 2 \mathcal{O}$ has linearly independent maximal minors. We claim that these maximal minors have a common linear factor. If this were not the case, then, as mentioned before, $\operatorname{Coker}\left(\varphi_{22}\right)$ would give a point in $\mathrm{M}_{\mathbb{P}^{3}}(3 m+2)$, and we would reach the contradictory conclusion that $2 \mathcal{O} \rightarrow F$ is the zero morphism. It is clear now that $\mathcal{K} \operatorname{er}\left(\varphi_{22}\right) \simeq \mathcal{O}(-2)$. The isomorphism $\varphi_{7}: \operatorname{Ker}\left(\varphi_{1}\right) \rightarrow \operatorname{Ker}\left(\varphi_{22}\right) / \mathcal{I} m\left(\varphi_{5}\right)$ shows that $q=1$. Resolving $\Omega^{1}$ in the previous sequence gives the resolution

$$
\begin{aligned}
0 & \rightarrow \mathcal{O}(-4) \oplus 3 \mathcal{O}(-3) \oplus \mathcal{O}(-2) \stackrel{\psi}{\rightarrow} 4 \mathcal{O}(-3) \oplus 6 \mathcal{O}(-2) \oplus 3 \mathcal{O}(-1) \\
& \stackrel{\varphi}{\rightarrow} 6 \mathcal{O}(-2) \oplus 2 \mathcal{O} \rightarrow F \rightarrow 0, \\
\psi & =\left[\begin{array}{ccc}
\psi_{11} & \psi_{12} & 0 \\
0 & \psi_{22} & 0 \\
0 & \psi_{32} & \psi_{33}
\end{array}\right], \quad \varphi=\left[\begin{array}{ccc}
\varphi_{11} & \varphi_{12} & 0 \\
0 & \varphi_{22} & \varphi_{23}
\end{array}\right],
\end{aligned}
$$




$$
\psi_{11}=\left[\begin{array}{c}
X \\
Y \\
Z \\
W
\end{array}\right], \quad \psi_{33}=\left[\begin{array}{c}
l_{1} \\
-l_{2} \\
l_{3}
\end{array}\right], \quad \varphi_{23}=\left[\begin{array}{lll}
l_{11} & l_{12} & l_{13} \\
l_{21} & l_{22} & l_{23}
\end{array}\right],
$$

where

$$
u l_{1}=\left|\begin{array}{ll}
l_{12} & l_{13} \\
l_{22} & l_{23}
\end{array}\right|, \quad u l_{2}=\left|\begin{array}{ll}
l_{11} & l_{13} \\
l_{21} & l_{23}
\end{array}\right|, \quad u l_{3}=\left|\begin{array}{ll}
l_{11} & l_{12} \\
l_{21} & l_{22}
\end{array}\right|
$$

for some $u \in V^{*}$. We claim that $\operatorname{rank}\left(\psi_{12}\right)=3$. To see this, we dualize the last exact sequence. According to [23, Lemma 3], we have the exact sequence

$$
\begin{aligned}
0 & \rightarrow 2 \mathcal{O}(-4) \oplus 6 \mathcal{O}(-2) \stackrel{\varphi^{\mathrm{T}}}{\rightarrow} 3 \mathcal{O}(-3) \oplus 6 \mathcal{O}(-2) \oplus 4 \mathcal{O}(-1) \\
& \stackrel{\psi^{\mathrm{T}}}{\rightarrow} \mathcal{O}(-2) \oplus 3 \mathcal{O}(-1) \oplus \mathcal{O} \rightarrow F^{\mathrm{D}} \rightarrow 0 .
\end{aligned}
$$

According to [23], $F^{\mathrm{D}}$ gives a point in $\mathrm{M}_{\mathbb{P}^{3}}(4 m-1)$. If $\psi_{12}$ were zero, then we would get a commutative diagram

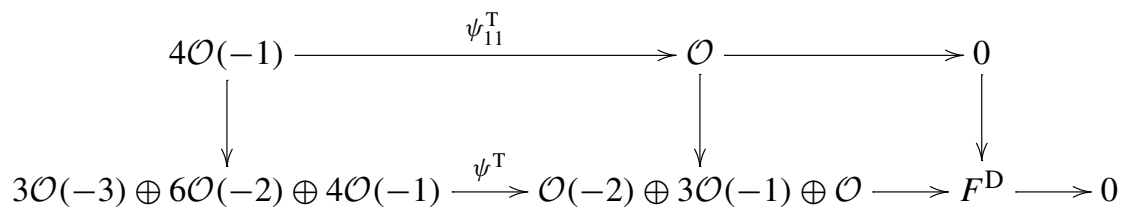

showing that the morphism $\mathcal{O} \rightarrow F^{\mathrm{D}}$ is zero. But the map $\mathrm{H}^{0}(\mathcal{O}) \rightarrow \mathrm{H}^{0}\left(F^{\mathrm{D}}\right)$ is injective because $\mathrm{H}^{0}\left(\operatorname{Coker}\left(\varphi^{\mathrm{T}}\right)\right)=0$. If $\operatorname{rank}\left(\psi_{12}\right)=1$, then the map $\mathcal{O} \rightarrow$ $F^{\mathrm{D}}$ would factor through the structure sheaf of a point, so it would be zero. If $\operatorname{rank}\left(\psi_{12}\right)=2$, then the map $\mathcal{O} \rightarrow F^{\mathrm{D}}$ would factor through the structure sheaf $\mathcal{O}_{L}$ of a line, so it would be zero because $\operatorname{Hom}\left(\mathcal{O}_{L}, F^{\mathrm{D}}\right)=0$. We reach again contradictions. This proves the claim.

Canceling $3 \mathcal{O}(-3)$, we get the resolution

$$
\begin{aligned}
0 & \rightarrow \mathcal{O}(-4) \oplus \mathcal{O}(-2) \rightarrow \mathcal{O}(-3) \oplus 6 \mathcal{O}(-2) \oplus 3 \mathcal{O}(-1) \\
& \stackrel{\varphi}{\rightarrow} 6 \mathcal{O}(-2) \oplus 2 \mathcal{O} \rightarrow F \rightarrow 0 .
\end{aligned}
$$

We have $\operatorname{rank}\left(\varphi_{12}\right)=6$ since otherwise $F$ would map surjectively to $\mathcal{O}_{H}(-2)$ for a plane $H \subset \mathbb{P}^{3}$. This is clearly impossible. Canceling $6 \mathcal{O}(-2)$, we finally get the resolution

$$
0 \rightarrow \mathcal{O}(-4) \oplus \mathcal{O}(-2) \stackrel{\psi}{\rightarrow} \mathcal{O}(-3) \oplus 3 \mathcal{O}(-1) \stackrel{\varphi}{\rightarrow} 2 \mathcal{O} \rightarrow F \rightarrow 0
$$

with

$$
\psi=\left[\begin{array}{cc}
l & 0 \\
f_{1} & l_{1} \\
f_{2} & -l_{2} \\
f_{3} & l_{3}
\end{array}\right], \quad \varphi=\left[\begin{array}{llll}
g_{1} & l_{11} & l_{12} & l_{13} \\
g_{2} & l_{21} & l_{22} & l_{23}
\end{array}\right] .
$$

The sheaf $E=\operatorname{Coker}\left(\varphi_{12}: 3 \mathcal{O}(-1) \rightarrow 2 \mathcal{O}\right)$ is supported on $H \cup\{P\}$, where $H$ is the plane given by the equation $u=0$, and $P$ is the point given by the ideal $\left\langle l_{1}, l_{2}, l_{3}\right\rangle$. Since $F$ is a quotient sheaf of $E$ and since $F$ has no zero-dimensional 
torsion, we see that $\operatorname{supp}(F) \subset H$. Applying the snake lemma to the commutative diagram in which the middle row is the dual of the last exact sequence

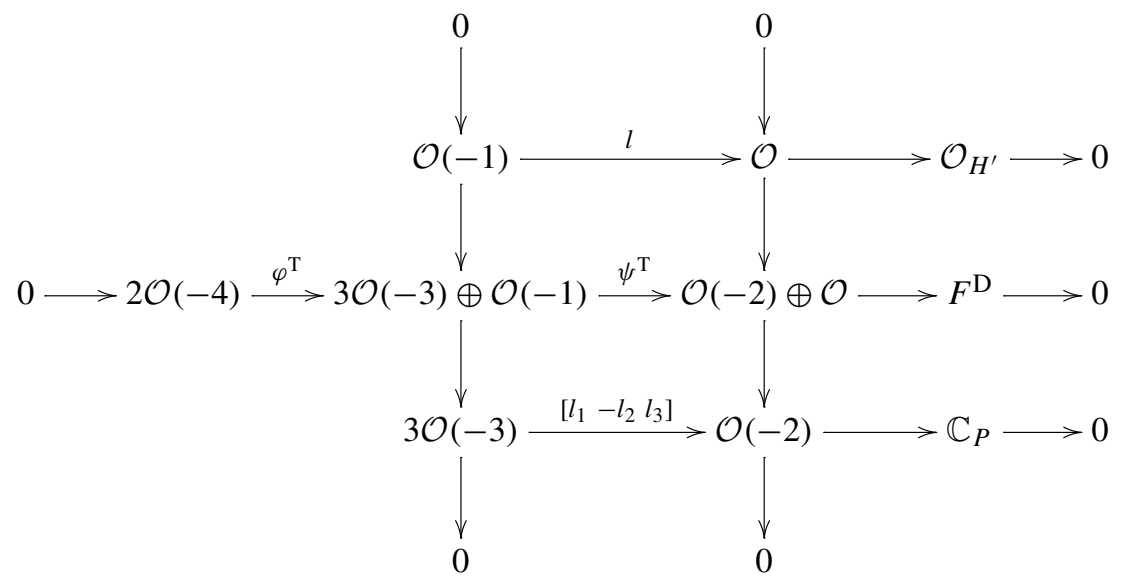

we get the exact sequence

$$
\mathcal{O}_{H^{\prime}} \rightarrow F^{\mathrm{D}} \rightarrow \mathbb{C}_{P} \rightarrow 0 .
$$

Since $F^{\mathrm{D}}$ has no zero-dimensional torsion, we see that $P \in H^{\prime}$, that is, $l \in$ $\operatorname{span}\left\{l_{1}, l_{2}, l_{3}\right\}$. Moreover, $\operatorname{supp}\left(F^{\mathrm{D}}\right) \subset H^{\prime}$, and hence also $\operatorname{supp}(F) \subset H^{\prime}$. It follows that $H=H^{\prime}$ since otherwise $F$ would be supported on a line, yet a vector bundle of rank greater than one on $\mathbb{P}^{1}$ is not stable. Thus, we may assume that $u=l$, and we may write

$$
\psi=\left[\begin{array}{cc}
l & 0 \\
f_{1} & l \\
f_{2} & -l_{2} \\
f_{3} & -l_{3}
\end{array}\right], \quad \varphi=\left[\begin{array}{llll}
g_{1} & l_{2} & l & 0 \\
g_{2} & l_{3} & 0 & l
\end{array}\right] .
$$

From the relations

$$
g_{1} l+l_{2} f_{1}+l f_{2}=0, \quad g_{2} l+l_{3} f_{1}+l f_{3}=0
$$

we see that $f_{1}$ is divisible by $l$. Performing column operations on $\psi$, we may assume that $f_{1}=0$. Thus, $g_{1}=-f_{2}, g_{2}=-f_{3}$. We have obtained resolution (19).

Conversely, given resolution (19), we also have resolution (20), and hence, by [11, Thm. 3.2.1], $F$ is semistable.

REMARK 6.2. The general sheaves in $\mathbf{R}$ and $\mathbf{E}$ have the same resolution of the form (17). The general sheaves in $\mathbf{P}$ and the sheaves in the wall-crossing have resolution (18). The stable sheaves $F$ with $\mathrm{h}^{0}(F)=2$ have resolution (19).

AcKnowledgments. We would like to thank Sheldon Katz and Han-Bom Moon for valuable discussions and comments. 


\section{References}

[1] D. Avritzer and I. Vainsencher, Compactifying the space of elliptic quartic curves, Complex projective geometry, London Math. Soc. Lecture Note Ser., 179, pp. 4758, Cambridge University Press, 1992.

[2] D. Chen, Mori's program for the Kontsevich moduli space $\bar{M}_{0,0}\left(\mathbb{P}^{3}, 3\right)$, Int. Math. Res. Not. IMRN (2008), article ID rnn067.

[3] D. Chen, I. Coskun, and C. Crissman, Towards Mori's program for the moduli space of stable maps, Amer. J. Math. 133 (2011), no. 5, 1389-1419.

[4] D. Chen and S. Nollet, Detaching embedded points, Algebra Number Theory 6 (2012), no. 4, 731-756.

[5] J. Choi and K. Chung, Moduli spaces of $\alpha$-stable pairs and wall-crossing on $\mathbb{P}^{2}$, J. Math. Soc. Japan 68 (2016), 685-709.

[6] _ Cohomology bounds for sheaves of dimension one, Internat. J. Math. 25 (2014), no. 11, 1450103.

[7] , The geometry of the moduli space of one-dimensional sheaves, Sci. China Math. 58 (2015), no. 3, 3487-3500.

[8] J. Choi and M. Maican, Torus action on the moduli spaces of plane sheaves, J. Geom. Phys. 83 (2014), 18-35.

[9] K. Chung and Y.-H. Kiem, Hilbert scheme of rational cubic curves via stable maps, Amer. J. Math. 133 (2011), no. 3, 797-834.

[10] K. Chung, J. Hong, and Y.-H. Kiem, Compactified moduli spaces of rational curves in projective homogeneous varieties, J. Math. Soc. Japan 64 (2012), no. 4, 1211-1248.

[11] J.-M. Drézet and M. Maican, On the geometry of the moduli spaces of semi-stable sheaves supported on plane quartics, Geom. Dedicata 152 (2011), 17-49.

[12] H.-G. Freiermuth and G. Trautmann, On the moduli scheme of stable sheaves supported on cubic space curves, Amer. J. Math. 126 (2004), 363-393.

[13] D. R. Grayson and M. E. Stillman, Macaulay2, a software system for research in algebraic geometry, available at 〈http://www.math.uiuc.edu/Macaulay2/〉.

[14] W. Fulton and R. Pandharipande, Notes on stable maps and quantum cohomology, Algebraic geometry—Santa Cruz, 1995, Proc. Sympos. Pure Math., 62, pp. 45-96, Amer. Math. Soc., Providence, RI, 1997.

[15] R. Hartshorne, Algebraic geometry, Grad. Texts in Math., 52, Springer-Verlag, 1977.

[16] _ The genus of space curves, Ann. Univ. Ferrara Sez. VII (N.S.) 40 (1994), 207-223.

[17] M. He, Espaces de modules de systèmes cohérents, Internat. J. Math. 7 (1998), 545598.

[18] D. Huybrechts, Fourier-Mukai transforms in algebraic geometry, Oxford Math. Monogr., Clarendon Press, 2006.

[19] D. Huybrechts and M. Lehn, The geometry of moduli spaces of sheaves, Aspects Math., E31, Vieweg, Braunschweig, 1997.

[20] O. Iena, Modification of Simpson moduli spaces of 1-dimensional sheaves by vector bundles. An experimental example, Ph.D. thesis, Technische Universität Kaiserslautern, Germany, 2009.

[21] J. Le Potier, Faisceaux semi-stables de dimension 1 sur le plan projectif, Rev. Roumaine Math. Pures Appl. 38 (1993), 635-678.

[22] __ Systèmes cohérents et structures de niveau, Astérisque, 214, 1993.

[23] M. Maican, A duality result for moduli spaces of semistable sheaves supported on projective curves, Rend. Semin. Mat. Univ. Padova 123 (2010), 55-68. 
[24] On the moduli spaces of semi-stable plane sheaves of dimension one and multiplicity five, Illinois J. Math. 55 (2011), 1467-1532.

[25] - The classification of semistable plane sheaves supported on sextic curves, Kyoto J. Math. 53 (2013), 739-786.

[26] J. McCleary, A user's guide to spectral sequences, Cambridge Stud. Adv. Math., 58, 2001.

[27] S. Nollet and E. Schlesinger, Hilbert schemes of degree four curves, Compos. Math. 139 (2003), 169-196.

[28] R. Pandharipande and R. P. Thomas, Curve counting via stable pairs in the derived category, Invent. Math. 178 (2009), 407-447.

[29] A. E. Parker, An elementary GIT construction of the moduli space of stable maps, Illinois J. Math. 51 (2007), no. 3, 1003-1025.

[30] C. T. Simpson, Moduli of representations of the fundamental group of a smooth projective variety. I, Publ. Math. Inst. Hautes Études Sci. 79 (1994), 47-129.

[31] J. Stoppa and R. P. Thomas, Hilbert schemes and stable pairs: GIT and derived category wall crossings, Bull. Soc. Math. France 139 (2011), no. 3, 297-339.

[32] M. Thaddeus, Stable pairs, linear systems and the Verlinde formula, Invent. Math. 177 (1994), no. 1, 317-353.

[33] R. P. Thomas, A holomorphic Casson invariant for Calabi-Yau 3-folds, and bundles on K3 fibrations, J. Differential Geom. 54 (2000), no. 2, 367-438.

[34] I. Tyomkin, On Severi varieties on Hirzebruch surfaces, Int. Math. Res. Not. IMRN (2007), no. 23, Art. ID rnm109, 31.

[35] C. Okonek, M. Schneider, and H. Spindler, Vector bundles on complex projective spaces, Progr. Math., 3, Birkhäuser, Boston, 1980.

[36] R. Piene and M. Schlessinger, On the Hilbert scheme compactification of the space of twisted cubics, Amer. J. Math. 107 (1985), 761-774.

[37] Y. Yuan, Moduli spaces of semistable sheaves of dimension 1 on $\mathbb{P}^{2}$, Pure Appl. Math. Q. 10 (2014), 723-766.

\section{J. Choi}

Department of Mathematics

Sookmyung Women's University

Seoul 04310

Korea

jwchoi@sookmyung.ac.kr

\author{
K. Chung \\ (corresponding author) \\ Department of Mathematics \\ Education \\ Kyungpook National University \\ 80 Daehakro \\ Bukgu, Daegu 41566 \\ Korea
}

krchung@knu.ac.kr

\author{
M. Maican \\ Institute of Mathematics \\ of the Romanian Academy \\ Calea Grivitei 21 \\ Bucharest 010702 \\ Romania
}

